\title{
Bifurcation Analysis for Nonlinear Recurrence Relations with Threshold Control and $2 k$-Periodic Coefficients
}

\author{
Liping Dou, ${ }^{1}$ Chengmin Hou, ${ }^{1}$ and Sui Sun Cheng ${ }^{2}$ \\ ${ }^{1}$ Department of Mathematics, Yanbian University, Yanji 133002, China \\ ${ }^{2}$ Department of Mathematics, Tsing Hua University, Hsinchu 30043, Taiwan \\ Correspondence should be addressed to Chengmin Hou; houchengmin@aliyun.com
}

Received 18 January 2015; Revised 10 March 2015; Accepted 11 March 2015

Academic Editor: Garyfalos Papashinopoulos

Copyright (C) 2015 Liping Dou et al. This is an open access article distributed under the Creative Commons Attribution License, which permits unrestricted use, distribution, and reproduction in any medium, provided the original work is properly cited.

A nonlinear recurrence involving a piecewise constant McCulloch-Pitts function and $2 k$-periodic coefficient sequences is investigated. By allowing the threshold parameter to vary from 0 to $\infty$, we work out a complete bifurcation analysis for the asymptotic behaviors of the corresponding solutions. Among other things, we show that each solution tends towards one of four different limits. Furthermore, the accompanying initial regions for each type of solutions can be determined. It is hoped that our analysis will provide motivation for further results for recurrent McCulloch-Pitts type neural networks.

\section{Introduction}

It is of great interest to see how an artificial neural network uses components which are closer to "biological" components. One particular important component can be described by means of the step (activation) function defined by

$$
f_{\lambda}(u)= \begin{cases}1, & \text { if } u \in(0, \lambda], \\ 0, & \text { if } u \in(-\infty, 0] \cup(\lambda,+\infty)\end{cases}
$$

with a nonnegative (threshold) real parameter $\lambda$. Roughly, the activation function mimics a so called McCulloch neuron that may receive an excitatory value (indicated by 1 ) if the input signal has strength within limits 0 and $\lambda$, and otherwise it remains intact with an inhibitory value (indicated by 0 ).

If we let $x_{n}$ be the state value of a neural unit during the time period $n$, then the recurrence relation

$$
x_{n}=F\left(f_{\lambda}\left(x_{n-1}\right), x_{n-2}\right)
$$

may be used to describe a one neuron McCulloch-Pitts system where the state value is updated from the two most recent state values.

Let $\mathbb{N}=\{0,1, \ldots\}$. Zhu and Huang [1] discussed the "limit cycles" of the recurrence relation

$$
x_{n}=a x_{n-2}+b f_{\lambda}\left(x_{n-1}\right), \quad n \in \mathbb{N},
$$

where $a \in(0,1), b \in(0,+\infty)$, and $f_{\lambda}: \mathbb{R} \rightarrow \mathbb{R}$ is defined by (1), in which the positive threshold $\lambda$ can be regarded as a bifurcation parameter. Then, Chen [2] considered the following recurrence relation:

$$
x_{n}=a_{n} x_{n-2}+b_{n} f_{\lambda}\left(x_{n-1}\right), \quad n \in \mathbb{N},
$$

where $\left\{a_{n}\right\}_{n=0}^{\infty}$ and $\left\{b_{n}\right\}_{n=0}^{\infty}$ are 2-periodic sequences with $a_{0}, a_{1} \in(0,1)$ and $b_{0}, b_{1} \in(0,+\infty)$. Asymptotic behaviors of these equations reflect their differences (see $[1,2])$. A good reason for studying (4) is that the constants $a$ and $b$ used in the physical model described by (3) may not be truly constant but exhibit fluctuating behaviors between two limits. Since in (4) we have chosen to consider the case where $a$ and $b$ are replaced by 2-periodic sequences, the question then arises as to what will happen if we choose general periodic sequences.

In this paper, we offer partial answers by considering the difference equation

$$
x_{n}=a_{n} x_{n-2}+b_{n} f_{\lambda}\left(x_{n-1}\right), \quad n \in \mathbb{N},
$$

where $\left\{a_{n}\right\}_{n=0}^{\infty}$ and $\left\{b_{n}\right\}_{n=0}^{\infty}$ are $2 k$-periodic sequences with $a_{i} \in(0,1), b_{i}=1-a_{i}$, and $i=0, \ldots, 2 k-1$. By studying this equation, we hope that the subsequent results will lead to much more general ones for complex systems involving similar periodic parameters and discontinuous controls. 
In order to study the asymptotic behavior of (5), let us first note that it is a three-term recurrence relation so that, given $x_{-2}$ and $x_{-1}$, we may calculate $x_{0}, x_{1}, x_{2}$, and so forth in a sequential manner. The resulting sequence $\left\{x_{n}\right\}_{n=-2}^{\infty}$ is naturally called a solution of (5). For example, when $\left\{a_{n}\right\}_{n=0}^{\infty}$ and $\left\{b_{n}\right\}_{n=0}^{\infty}$ are 4-periodic sequences, we may write

$$
\begin{aligned}
& x_{0}=a_{0} x_{-2}+b_{0} f_{\lambda}\left(x_{-1}\right), \\
& x_{1}=a_{1} x_{-1}+b_{1} f_{\lambda}\left(x_{0}\right), \\
& x_{2}=a_{2} x_{0}+b_{2} f_{\lambda}\left(x_{1}\right), \\
& x_{3}=a_{3} x_{1}+b_{3} f_{\lambda}\left(x_{2}\right), \\
& x_{4}=a_{4} x_{2}+b_{4} f_{\lambda}\left(x_{3}\right)=a_{0} x_{2}+b_{0} f_{\lambda}\left(x_{3}\right), \\
& x_{5}=a_{5} x_{3}+b_{5} f_{\lambda}\left(x_{4}\right)=a_{1} x_{3}+b_{1} f_{\lambda}\left(x_{4}\right),
\end{aligned}
$$

This motivates us to define a vector equation. Given a sequence $x=\left\{x_{n}\right\}_{n=a}^{\infty}$, its Casoratian vector sequence is $\left\{\left\langle x_{i}\right\rangle\right\}_{i=a}^{\infty}$, where $\left\langle x_{i}\right\rangle=\operatorname{col}\left(x_{i}, x_{i+1}, \ldots, x_{i+2 k-1}\right), i=a, a+$ $1, \ldots$. Then (5) is equivalent to the asynchronous vector equation

$$
\left\langle x_{2 k n}\right\rangle=A\left\langle x_{2 k n-2}\right\rangle+B \mathbf{f}_{\lambda}\left(\left\langle x_{2 k n-1}\right\rangle\right), \quad n=0,1,2, \ldots,
$$

where

$$
\begin{gathered}
A=\left(\begin{array}{cccc}
a_{0} & 0 & & 0 \\
0 & a_{1} & \ddots & \\
& \ddots & \ddots & 0 \\
0 & & 0 & a_{2 k-1}
\end{array}\right), \\
B=\left(\begin{array}{cccc}
b_{0} & 0 & & 0 \\
0 & b_{1} & \ddots & \\
& \ddots & \ddots & 0 \\
0 & & 0 & b_{2 k-1}
\end{array}\right), \\
\mathbf{f}_{\lambda}\left\langle x_{i}\right\rangle=\operatorname{col}\left(f_{\lambda}\left(x_{i}\right), f_{\lambda}\left(x_{i+1}\right), \ldots, f_{\lambda}\left(x_{i+2 k-1}\right)\right) .
\end{gathered}
$$

Note that, given $\left(x_{-2}, x_{-1}\right)$, we may use (7) to generate $\left\langle x_{0}\right\rangle,\left\langle x_{2 k}\right\rangle,\left\langle x_{4 k}\right\rangle, \ldots$ which, when "lined up," yields the same $x_{0}, x_{1}, x_{2}, \ldots$ as described above. For this reason, the sequence $\left\{\left\langle x_{i}\right\rangle\right\}_{i=0}^{\infty}$ will be called the solution of (7) determined by $\left(x_{-2}, x_{-1}\right)$.

Therefore, to obtain complete asymptotic behaviors of (5), we need to derive the behaviors of solutions of (7) determined by vectors $\left(x_{-2}, x_{-1}\right)$ in the entire plane.

The following result, however, can help us concentrate on solutions determined by vectors $\left(x_{-2}, x_{-1}\right)$ in $\mathbb{R}^{2} \backslash(-\infty, 0]^{2}$.

Theorem 1. A solution $\left\{\left\langle x_{2 k n}\right\rangle\right\}_{n=0}^{\infty}$ of (7) with $\left(x_{-2}, x_{-1}\right)$ in the nonpositive orthant $(-\infty, 0]^{2}$ is nonpositive and tends towards $\langle 0\rangle$.
Proof. Let $\left(x_{-2}, x_{-1}\right) \in(-\infty, 0]^{2}$. Then, by (7) and by induction, for any $n \in \mathbb{N}$ and $m \in\{0,1, \ldots, 2 k-1\}$, we may get $x_{2 k i+m} \in(-\infty, 0]$, that is,

$$
\left\langle x_{2 k n}\right\rangle=A\left\langle x_{2 k(n-1)}\right\rangle=\cdots=A^{n}\left\langle x_{0}\right\rangle .
$$

Since $\left\langle x_{0}\right\rangle \in(-\infty, 0]^{2 k}$ and $A^{2 n}$ tends towards 0 as $n$ tends towards $+\infty$, we see that $\left\langle x_{2 k n}\right\rangle$ tends towards $\langle 0\rangle$. The proof is complete.

Next, note that our system is autonomous (time invariant), and hence, if $\left\{\left\langle x_{2 k n}\right\rangle\right\}_{n=0}^{\infty}$ is a solution of (7), then, for any $m \in \mathbb{N}$, the sequence, $\left\langle y_{2 k n}\right\rangle$ defined by $\left\langle y_{2 k n}\right\rangle=\left\langle x_{2 k n+m}\right\rangle$ for $n=0,1, \ldots$, is also a solution of $(7)$.

Suppose $\left\{\left\langle x_{2 k n}\right\rangle\right\}^{\infty}$ is a solution of (7). Then, we say that

$\left\{\left\langle x_{2 k n}\right\rangle\right\}^{\infty}$ approaches a limit 1-cycle $\{\langle\alpha\rangle\}$ if $\lim _{i \rightarrow \infty}\left(x_{2 k i}, x_{2 k i+1}, \ldots, x_{2 k i+2 k-1}\right)=(\alpha, \alpha, \ldots, \alpha)$;

$\left\{\left\langle x_{2 k n}\right\rangle\right\}^{\infty}$ approaches a limit 2-cycle $\{\langle\alpha, \beta\rangle\}$ if $\alpha \neq \beta$ and $\lim _{i \rightarrow \infty}\left(x_{2 k i}, x_{2 k i+2}, \ldots, x_{2 k i+2 k-2}\right)=(\alpha, \alpha, \ldots, \alpha)$ and $\lim _{i \rightarrow \infty}\left(x_{2 k i+1}, x_{2 k i+3}, \ldots, x_{2 k i+2 k-1}\right)=(\beta, \beta, \ldots$, $\beta)$.

For the sake of convenience, we also need to introduce some notations. The numbers $\delta$ and $\rho$ are defined as

$$
\delta=a_{0} a_{2} \cdots a_{2 k-2}, \quad \rho=a_{1} a_{3} \cdots a_{2 k-1},
$$

while the numbers

$$
\begin{aligned}
& A_{i}^{\left(j_{0}, j_{1}, \ldots, j_{m}\right)}, B_{i}^{\left(j_{0}, j_{1}, \ldots, j_{m}\right)}, C_{i}^{\left(j_{0}, j_{1}, \ldots, j_{m}\right)}, \\
& D_{i}^{\left(j_{0}, j_{1}, \ldots, j_{m}\right)}, E_{i}^{\left(j_{0}, j_{1}, \ldots, j_{m}\right)}, G_{i}^{\left(j_{0}, j_{1}, \ldots, j_{m}\right)}, \\
& L_{i}^{\left(j_{0}, j_{1}, \ldots, j_{m}\right)}, M_{i}^{\left(j_{0}, j_{1}, \ldots, j_{m}\right)}, H_{i}^{\left(j_{0}, j_{1}, \ldots, j_{m}\right)}
\end{aligned}
$$

and their properties are listed in the Appendix. These numbers are introduced in order to break the plane into different parts such that the behavior of each solution of (7) which originates from each part may be traced.

In the sequel, we distinguish the cases (i) $\lambda>1$, (ii) $\lambda=$ 1 , and (iii) $0<\lambda<1$, and then, for different $\left(x_{-2}, x_{-1}\right) \in$ $\mathbb{R}^{2} \backslash(-\infty, 0]^{2}$, we find the asymptotic behaviors of the corresponding solutions.

\section{The Case $\lambda>1$}

We begin with the following.

Lemma 2. Let $\lambda>1$. If $\left\{\left\langle x_{2 k n}\right\rangle\right\}_{n=0}^{\infty}$ is a solution of (7) with $\left(x_{-2}, x_{-1}\right) \in \mathbb{R}^{2} \backslash(-\infty, 0]^{2}$, then there exist integers $r \in$ $\{0,1, \ldots, 2 k-2\}$ and $j \in \mathbb{N}$ such that $0<x_{2 k j+r}, x_{2 k j+r+1} \leq \lambda$.

Proof. From our assumption, we have $a_{i} \lambda+b_{i}<\lambda$ for $i=0,1, \ldots, 2 k-1$. Let $\left\{\left\langle x_{2 k n}\right\rangle\right\}_{n=0}^{\infty}$ be a solution of (7) with $\left(x_{-2}, x_{-1}\right) \in \mathbb{R}^{2} \backslash(-\infty, 0]^{2}$. Then, there are eight cases. 
Case 1. If $\left(x_{-2}, x_{-1}\right) \in(0, \lambda]^{2}$, then by (7) we may easily obtain $\left(x_{0}, x_{1}\right) \in(0, \lambda]^{2}$, our assertion is true by taking $j=0$ and $r=0$.

Case 2. Suppose $\left(x_{-2}, x_{-1}\right) \in(0, \lambda] \times(\lambda,+\infty)$. Then by (7) and induction we may see that $x_{2 k i+2 m} \in(0, \lambda]$ for any $m \in$ $\{1,2, \ldots, k-1\}$ and $i \in \mathbb{N}$; and hence,

$$
\begin{aligned}
x_{2 k i+2 m-1}= & a_{2 m-1} x_{2 k i+2 m-3}+b_{2 m-1} \\
= & a_{1} a_{3} \cdots a_{2 m-3} \rho^{i} x_{-1}+1 \\
& -a_{1} a_{3} \cdots a_{2 m-3} \rho^{i}, \quad m \in\{1,2, \ldots, k\} .
\end{aligned}
$$

Thus, $\lim _{i \rightarrow \infty} x_{2 k i+2 m-1}=1 \in(0, \lambda]$ for any $m \in\{1,2, \ldots, k\}$. Then, there exists enough large $j \in \mathbb{N}$ such that $\left(x_{2 k j}, x_{2 k j+1}\right) \in$ $(0, \lambda]^{2}$.

Case 3. Suppose $\left(x_{-2}, x_{-1}\right) \in(\lambda,+\infty) \times(0, \lambda]$. As in Case 2, we may show that there exists enough large $j \in \mathbb{N}$ such that $\left(x_{2 k j}, x_{2 k j+1}\right) \in(0, \lambda]^{2}$. Our assertion is thus true.

Case 4. Suppose $\left(x_{-2}, x_{-1}\right) \in(\lambda,+\infty) \times(\lambda,+\infty)$. We may first show that there is a $j \in\{0,1, \ldots\}$ such that $x_{j} \in(0, \lambda]$. Otherwise, for any $j \in\{0,1, \ldots\}$, we have $x_{j} \in(\lambda,+\infty)$. Then by (7) we have

$$
\left\langle x_{2 k n}\right\rangle=A\left\langle x_{2 k(n-1)}\right\rangle=\cdots=A^{n}\left\langle x_{0}\right\rangle \text {. }
$$

Since $\left\langle x_{0}\right\rangle \in(0, \infty)^{2 k}$ and $A^{2 n}$ tends towards 0 as $n$ tends towards $+\infty$, we see that $\left\langle x_{2 k n}\right\rangle$ tends towards $\langle 0\rangle$, which is a contradiction. The rest of the proof comes from the assertions in Cases 2 and 3.

Case 5. Suppose $\left(x_{-2}, x_{-1}\right) \in(-\infty, 0] \times(0, \lambda]$. Then by (7) and induction we may see that $x_{2 k i+2 m-1} \in(0, \lambda]$ for any $m \in$ $\{1,2, \ldots, k\}$ and $i \in \mathbb{N}$; and hence,

$$
\begin{aligned}
x_{2 k i+2 m-2}= & a_{2 m-2} x_{2 k i+2 m-2}+b_{2 m-2} f_{\lambda}\left(x_{2 k i-1}\right) \\
= & a_{0} a_{2} \cdots a_{2 m-2} \delta^{i} x_{-2}+1 \\
& -a_{0} a_{2} \cdots a_{2 m-2} \delta^{i}, \quad m \in\{1,2, \ldots\} .
\end{aligned}
$$

Thus, $\lim _{i \rightarrow \infty} x_{2 k i+2 m-2}=1 \in(0, \lambda]$ for any $m \in\{1,2, \ldots, k\}$. Then, there exists enough large $j \in \mathbb{N}$ such that $\left(x_{2 k j}, x_{2 k j+1}\right) \in$ $(0, \lambda]^{2}$.

Case 6. Suppose $\left(x_{-2}, x_{-1}\right) \in(0, \lambda] \times(-\infty, 0]$. As in Case 5 , we may show that there exists enough large $j \in \mathbb{N}$ such that $\left(x_{2 k j}, x_{2 k j+1}\right) \in(0, \lambda]^{2}$. Our assertion is thus true.

Case 7. Suppose $\left(x_{-2}, x_{-1}\right) \in(\lambda,+\infty) \times(-\infty, 0]$. In view of (A.3), there exist $m \in\{0,1, \ldots, k-1\}$ and $i \in \mathbb{N}$ such that $\left(x_{-2}, x_{-1}\right) \in\left(D_{i}^{(0,2, \ldots, 2 m-2)}, D_{i}^{(0,2, \ldots, 2 m)}\right] \times(-\infty, 0]$. Next, we consider two cases.
(I) Consider $i=0, m \geq 0$. Then by (7) we have

$$
\begin{aligned}
x_{0}= & a_{0} x_{-2}+b_{0} f_{\lambda}\left(x_{-1}\right) \\
& =a_{0} x_{-2} \in\left(D_{0}^{(2, \ldots, 2 m-2)}, D_{0}^{(2, \ldots, 2 m)}\right], \\
x_{1} & =a_{1} x_{-1}+b_{1} f_{\lambda}\left(x_{0}\right)=a_{1} x_{-1} \in(-\infty, 0], \\
& \vdots \\
x_{2 m-1}= & a_{2 m-1} x_{2 m-2}+b_{2 m-1} f_{\lambda}\left(x_{2 m-1}\right) \\
& =a_{1} a_{3} \cdots a_{2 m-1} x_{-1} \in(-\infty, 0], \\
x_{2 m}= & a_{2 m} x_{2 m-2}+b_{2 m} f_{\lambda}\left(x_{2 m-1}\right) \\
& =a_{0} a_{2} \cdots x_{-2} \in\left(a_{2 m} D_{0}^{(-2)}, D_{0}^{(-2)}\right] \subset(0, \lambda],
\end{aligned}
$$

that is, $\left(x_{2 m-1}, x_{2 m}\right) \in(-\infty, 0] \times(0, \lambda]$. Our assertion is true by Case 6.

(II) Consider $i>0, m \geq 0$. Then by (7) we have

$$
\begin{aligned}
x_{0} & =a_{0} x_{-2}+b_{0} f_{\lambda}\left(x_{-1}\right) \\
& =a_{0} x_{-2} \in\left(D_{i}^{(2, \ldots, 2 m-2)}, D_{i}^{(2, \ldots, 2 m)}\right], \\
x_{1} & =a x_{-1}+b_{1} f_{\lambda}\left(x_{0}\right)=a_{1} x_{-1} \in(-\infty, 0], \\
& \vdots \\
x_{2 k-2} & =a_{2 k-2} x_{2 k-4}+b_{2 k-2} f_{\lambda}\left(x_{2 k-3}\right) \\
& =\delta x_{-2} \in\left(D_{i-1}^{(0,2, \ldots, 2 m-2)}, D_{i-1}^{(0,2, \ldots, 2 m)}\right], \\
x_{2 k-1} & =a_{2 k-1} x_{2 k-3}+b_{2 k-1} f_{\lambda}\left(x_{2 k-2}\right) \\
& =\rho x_{-1} \in(-\infty, 0]
\end{aligned}
$$

and, by induction, we have

$$
\begin{aligned}
x_{2 k i} & =a_{0} x_{2 k i-2}+b_{0} f_{\lambda}\left(x_{2 k i-1}\right) \\
& =a_{0} \delta^{i} x_{-2} \in\left(D_{0}^{(2, \ldots, 2 m-2)}, D_{0}^{(2, \ldots, 2 m)}\right], \\
x_{2 k i+1} & =a_{1} x_{2 k i-1}+b_{1} f_{\lambda}\left(x_{2 k i}\right) \\
& =a_{1} \rho^{i} x_{-1} \in(-\infty, 0],
\end{aligned}
$$

$$
\begin{aligned}
x_{2 k i+2 m-1} & =a_{2 m-1} x_{2 k i+2 m-3}+b_{2 m-1} f_{\lambda}\left(x_{2 k i+2 m-2}\right) \\
& =a_{1} a_{3} \cdots a_{2 m-1} \rho^{i} x_{-1} \in(-\infty, 0], \\
x_{2 k i+2 m} & =a_{2 m} x_{2 k i+2 m-2}+b_{2 m} f_{\lambda}\left(x_{2 k i+2 m-1}\right) \\
& =a_{0} a_{2} \cdots a_{2 m} \delta^{i} x_{-2} \in\left(a_{2 m} D_{0}^{(-2)}, D_{0}^{(-2)}\right] \subset(0, \lambda],
\end{aligned}
$$

that is, $\left(x_{2 k i+2 m-1}, x_{2 k i+2 m}\right) \in(-\infty, 0] \times(0, \lambda]$. Our assertion is true by means of Case 6 . 
Case 8. Suppose $\left(x_{-2}, x_{-1}\right) \in(-\infty, 0] \times(\lambda,+\infty)$. Then, there exist $m \in\{0,1, \ldots, k-1\}$ and $i \in \mathbb{N}$ such that $\left(x_{-2}, x_{-1}\right) \in(-\infty, 0] \times\left(E_{i}^{(1,3, \ldots, 2 m-1)}, E_{i}^{(1,3, \ldots, 2 m+1)}\right]$. As in Case 7 , we may show that $\left(x_{2 k i+2 m}, x_{2 k i+2 m+1}\right) \in(-\infty, 0] \times$ $(0, \lambda]$. Our assertion is true by means of Case 5 .

Theorem 3. Let $\lambda>1$. If $\left\{\left\langle x_{2 k n}\right\rangle\right\}_{n=0}^{\infty}$ is a solution of (7) with $\left(x_{-2}, x_{-1}\right) \in \mathbb{R}^{2} \backslash(-\infty, 0]^{2}$, then it approaches the limit 1-cycle $\langle 1\rangle$.

Proof. By Lemma 2, we may assume without loss of generality that $\left(x_{-2}, x_{-1}\right) \in(0, \lambda]^{2}$. Then, by (7) and induction, $x_{2 k i+j}=$ $a_{j} x_{2 k i+j-2}+b_{j} \in(0, \lambda]$ for $i \in \mathbb{N}$ and $j \in\{0,1, \ldots, 2 k-1\}$; that is,

$$
\begin{aligned}
\left\langle x_{2 k n}\right\rangle & =A\left\langle x_{2 k(n-1)}\right\rangle+B=\cdots \\
& =A^{n}\left\langle x_{0}\right\rangle+A^{n-1} B+A^{n-2} B+\cdots+B \\
& =A^{n}\left\langle x_{0}\right\rangle+E-A^{i} .
\end{aligned}
$$

Since $\left\langle x_{0}\right\rangle \in(-\infty, 0]^{2 k}$ and $A^{2 n}$ tends towards 0 as $n$ tends towards $+\infty$, we see that $\left\{\left\langle x_{2 k n}\right\rangle\right\}$ tends towards the limit 1cycle $\langle 1\rangle$. The proof is complete.

\section{The Case $\lambda=1$}

In this section, we assume $\lambda=1$. Set

$$
\begin{gathered}
\Psi=(0, \lambda]^{2} \cup(-\infty, 0] \times(0, \lambda] \cup(0, \lambda] \times(-\infty, 0] \\
\cup(-\infty, 0] \times(\lambda, \infty) \cup(\lambda, \infty) \times(-\infty, 0], \\
\Omega=(0, \lambda] \times(\lambda, \infty) \cup(\lambda, \infty) \times(0, \lambda] \cup(\lambda, \infty) \times(\lambda, \infty) .
\end{gathered}
$$

Note that a simple plot of the regions $\Psi$ and $\Omega$ in the plane shows that they are disjoint and form the complement of the region $(-\infty, \lambda]^{2}$.

Lemma 4. Let $\lambda=1$. If $\left\{\left\langle x_{2 k n}\right\rangle\right\}_{n=0}^{\infty}$ is a solution of (7) with $\left(x_{-2}, x_{-1}\right) \in \Psi$, then there exist integer $r \in\{0,1, \ldots, 2 k-2\}$ and $j \in \mathbb{N}$ such that $0<x_{2 k j+r}, x_{2 k j+r+1} \leq \lambda$.

The proof is similar to those discussed in Cases 5 through 8 in the proof of Lemma 2 and, hence, is skipped.

Theorem 5. Let $\lambda=1$. If $\left\{\left\langle x_{2 k n}\right\rangle\right\}_{n=0}^{\infty}$ is a solution of (7) with $\left(x_{-2}, x_{-1}\right) \in \Psi$, then it approaches the limit 1-cycle $\langle 1\rangle$.

Proof. By Lemma 4, we may assume without loss of generality that $\left(x_{-2}, x_{-1}\right) \in(0, \lambda]^{2}$. Then, similar to the proof of Theorem 3, we have $x_{2 k i+j}=a_{j} x_{2 k i+j-2}+b_{j} \in(0, \lambda]$ for $i \in \mathbb{N}$ and $j \in\{0,1, \ldots, 2 k-1\}$; that is,

$$
\begin{aligned}
\left\langle x_{2 k n}\right\rangle & =A\left\langle x_{2 k(n-1)}\right\rangle+B=\cdots \\
& =A^{n}\left\langle x_{0}\right\rangle+A^{n-1} B+A^{n-2} B+\cdots+B \\
& =A^{n}\left\langle x_{0}\right\rangle+E-A^{i} .
\end{aligned}
$$

Since $\left\langle x_{0}\right\rangle \in(-\infty, 0]^{2 k}$ and $A^{2 n}$ tends towards 0 as $n$ tends towards $+\infty$, we see that $\left\{\left\langle x_{2 k n}\right\rangle\right\}$ tends towards the limit 1cycle $\langle 1\rangle$. The proof is complete.

Theorem 6. A solution $\left\{\left\langle x_{2 k n}\right\rangle\right\}_{n=0}^{\infty}$ of (7) with $\left(x_{-2}, x_{-1}\right) \in$ $(0, \lambda] \times(\lambda,+\infty)$ tends towards the limit 2 -cycle $\langle 0,1\rangle$.

Proof. By (7), we have

$$
\begin{aligned}
x_{0}= & a_{0} x_{-2}+b_{0} f_{\lambda}\left(x_{-1}\right)=a_{0} x_{-2} \in(0, \lambda], \\
x_{1}= & a_{1} x_{-1}+b_{1} f_{\lambda}\left(x_{0}\right)=a_{1} x_{-1}+b_{1}>\lambda, \\
& \vdots \\
x_{2 k-2}= & a_{2 k-2} x_{2 k-4}+b_{2 k-2} f_{\lambda}\left(x_{2 k-3}\right) \\
= & \delta x_{-2} \in(0, \lambda], \\
x_{2 k-1} & =a_{2 k-1} x_{2 k-3}+b_{2 k-1} f_{\lambda}\left(x_{2 k-2}\right) \\
& =\rho x_{-1}+1-\rho>\lambda,
\end{aligned}
$$

and by induction,

$$
\begin{aligned}
x_{2 k n} & =a_{0} x_{2 k n-2}+b_{0} f_{\lambda}\left(x_{2 k n-1}\right) \\
& =a_{0} \delta^{n} x_{-2} \in(0, \lambda], \\
x_{2 k n+1} & =a_{1} x_{2 k n-1}+b_{1} f_{\lambda}\left(x_{2 k n}\right) \\
& =a_{1} \rho^{n} x_{-1}+1-a_{1} \rho^{n}>\lambda, \\
& \vdots \\
x_{2 k n+2 k-1} & =a_{2 k-1} x_{2 k n+2 k-3}+b_{2 k-1} f_{\lambda}\left(x_{2 k n+2 k-2}\right) \\
& =\rho^{n+1} x_{-1}+1-\rho^{n+1}>\lambda .
\end{aligned}
$$

Therefore, $\left\{\left\langle x_{2 k n}\right\rangle\right\}$ tends towards the limit 2-cycle $\langle 0,1\rangle$.

Theorem 7. A solution $\left\{\left\langle x_{2 k n}\right\rangle\right\}_{n=0}^{\infty}$ of (7) with $\left(x_{-2}, x_{-1}\right) \in$ $(\lambda,+\infty) \times(0, \lambda]$ tends towards the limit 2 -cycle $\langle 1,0\rangle$.

The proof is similar to Theorem 6 and is skipped.

Theorem 8. Suppose that $\left\{\left\langle x_{2 k n}\right\rangle\right\}_{n=0}^{\infty}$ is a solution of (7) with $\left(x_{-2}, x_{-1}\right) \in\left(D_{i}^{(0,2, \ldots, 2 m-2)}, D_{i}^{(0,2, \ldots, 2 m)}\right] \times\left(E_{j}^{(1,3, \ldots, 2 l-1)}\right.$, $\left.E_{j}^{(1,3, \ldots, 2 l+1)}\right]$, where $i, j \in\{0,1, \ldots\}$ and $m, l \in\{0,1,2, \ldots, k-1\}$. Then,

(i) $\lim _{n \rightarrow \infty}\left\langle x_{2 k n}\right\rangle=\langle 0,1\rangle$ for $i=j, m \leq l$;

(ii) $\lim _{n \rightarrow \infty}\left\langle x_{2 k n}\right\rangle=\langle 1,0\rangle$ for $i=j, m>l$;

(iii) $\lim _{n \rightarrow \infty}\left\langle x_{2 k n}\right\rangle=\langle 1,0\rangle$ for $i<j$;

(iv) $\lim _{n \rightarrow \infty}\left\langle x_{2 k n}\right\rangle=\langle 0,1\rangle$ for $i>j$.

Proof. Suppose that $\left(x_{-2}, x_{-1}\right) \in\left(D_{i}^{(0,2, \ldots, 2 m-2)}, D_{i}^{(0,2, \ldots, 2 m)}\right] \times$ $\left(E_{j}^{(1,3, \ldots, 2 l-1)}, E_{j}^{(1,3, \ldots, 2 l+1)}\right]$. 
(i) We distinguish two different cases.

Case 1. Consider $0=i=j, 0 \leq m \leq l$. Then,

$$
\begin{aligned}
x_{0} & =a_{0} x_{-2}+b_{0} f_{\lambda}\left(x_{-1}\right) \\
& =a_{0} x_{-2} \in\left(D_{0}^{(2, \ldots, 2 m-2)}, D_{0}^{(2, \ldots, 2 m)}\right], \\
x_{1} & =a_{1} x_{-1}+b_{1} f_{\lambda}\left(x_{0}\right) \\
& =a_{1} x_{-1} \in\left(E_{0}^{(3, \ldots, 2 l-1)}, E_{0}^{(3, \ldots, 2 l+1)}\right], \\
& \vdots \\
x_{2 m} & =a_{2 m} x_{2 m-2}+b_{2 m} f_{\lambda}\left(x_{2 m-1}\right) \\
& =a_{1} a_{3} \cdots a_{2 m} \in\left(a_{2 m} D_{0}^{(-2)}, D_{0}^{(-2)}\right] \subset(0, \lambda], \\
x_{2 m+1} & =a_{2 m+1} x_{2 m-2}+b_{2 m+1} f_{\lambda}\left(x_{2 m-1}\right) \\
& >a_{2 m+1} \lambda+b_{2 m+1}>\lambda .
\end{aligned}
$$

Case 2. Consider $0<i=j, 0 \leq m \leq l$. Then,

$$
\begin{aligned}
x_{0}= & a_{0} x_{-2}+b_{0} f_{\lambda}\left(x_{-1}\right) \\
= & a_{0} x_{-2} \in\left(D_{i}^{(2, \ldots, 2 m-2)}, D_{i}^{(2, \ldots, 2 m)}\right], \\
x_{1}= & a_{1} x_{-1}+b_{1} f_{\lambda}\left(x_{0}\right) \\
= & a_{1} x_{-1} \in\left(E_{j}^{(3, \ldots, 2 l-1)}, E_{j}^{(3, \ldots, 2 l+1)}\right], \\
& \vdots \\
x_{2 k-2}= & a_{2 k-2} x_{2 k-4}+b_{2 k-2} f_{\lambda}\left(x_{2 k-3}\right) \\
= & a_{0} a_{2} \cdots a_{2 k-2} x_{-2} \in\left(D_{i-1}^{(0,2, \ldots, 2 m-2)}, D_{i-1}^{(0,2, \ldots, 2 m)}\right], \\
= & a_{2 k-1} x_{2 k-3}+b_{2 k-1} f_{\lambda}\left(x_{2 k-2}\right) \\
= & \rho x_{-1} \in\left(E_{j-1}^{(1,3, \ldots, 2 l-1)}, E_{j-1}^{(1,3, \ldots, 2 l+1)}\right],
\end{aligned}
$$

and by induction, we have

$$
\begin{aligned}
x_{2 k i}= & a_{0} x_{2 k i-2}+b_{0} f_{\lambda}\left(x_{2 k i-1}\right) \\
= & a_{0} x_{2 k i-2} \in\left(D_{0}^{(2, \ldots, 2 m-2)}, D_{0}^{(2, \ldots, 2 m)}\right], \\
x_{2 k i+1}= & a_{1} x_{2 k i-1}+b_{1} f_{\lambda}\left(x_{2 k i}\right) \\
= & a_{1} x_{2 k i-1} \in\left(E_{0}^{(3, \ldots, 2 l-1)}, E_{0}^{(3, \ldots, 2 l+1)}\right], \\
\vdots & \\
x_{2 k i+2 m-1} & a_{2 m-1} x_{2 k i+2 m-3}+b_{2 m-1} f_{\lambda}\left(x_{2 k i+2 m-2}\right) \\
= & a_{1} a_{3} \cdots a_{2 m-1} \rho^{i} x_{-1} \\
& \in\left(E_{j-i}^{(2 m+1, \ldots, 2 l+1)}, E_{j-i}^{(2 m+1, \ldots, 2 l+1)}\right], \\
= & a_{2 m} x_{2 k i+2 m-2}+b_{2 m} f_{\lambda}\left(x_{2 k i+2 m-1}\right) \\
= & a_{1} a_{3} \cdots a_{2 m} \delta^{i} \in\left(a_{2 m} D_{0}^{(-2)}, D_{0}^{(-2)}\right] \subset(0, \lambda] .
\end{aligned}
$$

(ii) Similar to (i), by distinguishing two different cases and by induction, we have the following.

Case 1. Consider $\left(x_{2 l}, x_{2 l+1}\right) \in(\lambda,+\infty) \times(0, \lambda], 0=i=j, 0 \leq$ $l \leq m$.

Case 2. Consider $\left(x_{2 k i+2 l+1}, x_{2 k i+2 l+2}\right) \in(0, \lambda] \times(\lambda,+\infty), 0<$ $i=j, 0 \leq l<m$.

(iii) We distinguish four different cases.

Case 1. Consider $j>i=0, m \geq l \geq 0$. By (7),

$$
\begin{aligned}
x_{0} & =a_{0} x_{-2}+b_{0} f_{\lambda}\left(x_{-1}\right) \\
& =a_{0} x_{-2} \in\left(D_{0}^{(2, \ldots, 2 m-2)}, D_{0}^{(2 \ldots, 2 m)}\right], \\
x_{1} & =a_{1} x_{-1}+b_{1} f_{\lambda}\left(x_{0}\right) \\
& =a_{1} x_{-1} \in\left(E_{j}^{(3, \ldots, 2 l-1)}, E_{j}^{(3, \ldots, 2 l+1)}\right], \\
& \vdots \\
x_{2 m} & =a_{2 m} x_{2 m-2}+b_{2 m} f_{\lambda}\left(x_{2 m-1}\right) \\
& =a_{2 m} x_{2 m-2} \in\left(a_{2 m} D_{0}^{(-2)}, D_{0}^{(-2)}\right] \subset(0, \lambda], \\
x_{2 m+1} & =a_{2 m+1} x_{2 m-1}+b_{2 m+1} f_{\lambda}\left(x_{2 m}\right) \\
& =a_{2 m+1} x_{2 m-1}+b_{2 m+1}>\lambda .
\end{aligned}
$$

Case 2. Consider $j>i=0, l \geq m \geq 0$. Then, by (7),

$$
\begin{aligned}
x_{0} & =a_{0} x_{-2}+b_{0} f_{\lambda}\left(x_{-1}\right) \\
& =a_{0} x_{-2} \in\left(D_{0}^{(2, \ldots, 2 m-2)}, D_{0}^{(2, \ldots, 2 m)}\right], \\
x_{1} & =a_{1} x_{-1}+b_{1} f_{\lambda}\left(x_{0}\right) \\
& =a_{1} x_{-1} \in\left(E_{j}^{(3, \ldots, 2 l-1)}, E_{j}^{(3, \ldots, 2 l+1)}\right], \\
& \vdots \\
x_{2 m} & =a_{2 m} x_{2 m-2}+b_{2 m} f_{\lambda}\left(x_{2 m-1}\right) \\
& =a_{2 m} x_{2 m-2} \in\left(a_{2 m} D_{0}^{(-2)}, D_{0}^{(-2)}\right] \subset(0, \lambda], \\
x_{2 m+1} & =a_{2 m+1} x_{2 m-1}+b_{2 m+1} f_{\lambda}\left(x_{2 m}\right) \\
& =a_{2 m+1} x_{2 m-1}+b_{2 m+1}>\lambda .
\end{aligned}
$$

Case 3. Consider $j>i>0, m \geq l \geq 0$. Then,

$$
\begin{aligned}
x_{0}= & a_{0} x_{-2}+b_{0} f_{\lambda}\left(x_{-1}\right) \\
& =a_{0} x_{-2} \in\left(D_{i}^{(2, \ldots, 2 m-2)}, D_{i}^{(2, \ldots, 2 m)}\right], \\
x_{1} & =a_{1} x_{-1}+b_{1} f_{\lambda}\left(x_{0}\right) \\
& =a_{1} x_{-1} \in\left(E_{j}^{(3, \ldots, 2 l-1)}, E_{j}^{(3, \ldots, 2 l+1)}\right], \\
& \vdots \\
x_{2 k-2}= & a_{2 k-2} x_{2 k-4}+b_{2 k-2} f_{\lambda}\left(x_{2 k-3}\right) \\
= & a_{0} a_{2} \cdots a_{2 k-2} x_{-2} \in\left(D_{i-1}^{(0,2, \ldots, 2 m-2)}, D_{i-1}^{(0,2, \ldots, 2 m)}\right], \\
x_{2 k-1} & a_{2 k-1} x_{2 k-3}+b_{2 k-1} f_{\lambda}\left(x_{2 k-2}\right) \\
= & a_{1} a_{3} \cdots a_{2 k-1} x_{-1} \in\left(E_{j-1}^{(1,3, \ldots, 2 l-1)}, E_{j-1}^{(1,3, \ldots, 2 l+1)}\right],
\end{aligned}
$$


and, by induction, we have

$$
\begin{aligned}
x_{2 k i}= & a_{0} x_{2 k i-2}+b_{0} f_{\lambda}\left(x_{2 k i-1}\right) \\
= & a_{0} x_{2 k i-2} \in\left(D_{0}^{(2, \ldots, 2 m-2)}, D_{0}^{(2, \ldots, 2 m)}\right], \\
x_{2 k i+1}= & a_{1} x_{2 k i-1}+b_{1} f_{\lambda}\left(x_{2 k i}\right) \\
= & a_{1} x_{2 k i-1} \in\left(E_{j-i}^{(3, \ldots, 2 l-1)}, E_{j-i}^{(3, \ldots, 2 l+1)}\right], \\
\vdots & \\
x_{2 k i+2 m-1}= & a_{2 m-1} x_{2 k i+2 m-3} \\
& +b_{2 m-1} f_{\lambda}\left(x_{2 k i+2 m-2}\right) \\
= & a_{1} a_{3} \cdots a_{2 m-1} \rho^{i} x_{-1} \\
& \in\left(E_{j-i}^{(1,3, \ldots, 2 l-1,2 m+1, \ldots, 2 k-1)}, E_{j-i}^{(1,3, \ldots, 2 l+1,2 m+1, \ldots, 2 k-1)}\right], \\
= & a_{2 m} x_{2 k i+2 m-2}+b_{2 m} f_{\lambda}\left(x_{2 k i+2 m-1}\right) \\
= & a_{0} a_{2} \cdots a_{2 m} \delta^{i} x_{-2} \in\left(a_{2 m} D_{0}^{(-2)}, D_{0}^{(-2)}\right] \subset(0, \lambda] .
\end{aligned}
$$

Case 4. Consider $j>i>0, l \geq m \geq 0$. Then,

$$
\begin{aligned}
x_{0} & =a_{0} x_{-2}+b_{0} f_{\lambda}\left(x_{-1}\right) \\
& =a_{0} x_{-2} \in\left(D_{i}^{(2, \ldots, 2 m-2)}, D_{i}^{(2, \ldots, 2 m)}\right], \\
x_{1} & =a_{1} x_{-1}+b_{1} f_{\lambda}\left(x_{0}\right) \\
& =a_{1} x_{-1} \in\left(E_{j}^{(3, \ldots, 2 l-1)}, E_{j}^{(3, \ldots, 2 l+1)}\right], \\
& \vdots \\
x_{2 k-2} & =a_{2 k-2} x_{2 k-4}+b_{2 k-2} f_{\lambda}\left(x_{2 k-3}\right) \\
& =a_{0} a_{2} \cdots a_{2 k-2} x_{-2} \in\left(D_{i-1}^{(0,2, \ldots, 2 m-2)}, D_{i-1}^{(0,2, \ldots, 2 m)}\right], \\
x_{2 k-1} & =a_{2 k-1} x_{2 k-3}+b_{2 k-1} f_{\lambda}\left(x_{2 k-2}\right) \\
& =a_{1} a_{3} \cdots a_{2 k-1} x_{-1} \in\left(E_{j-1}^{(1,3, \ldots, 2 l-1)}, E_{j-1}^{(1,3, \ldots, 2 l+1)}\right]
\end{aligned}
$$

and, by induction, we have

$$
\begin{aligned}
x_{2 k i}= & a_{0} x_{2 k i-2}+b_{0} f_{\lambda}\left(x_{2 k i-1}\right) \\
= & a_{0} x_{2 k i-2} \in\left(D_{0}^{(2, \ldots, 2 m-2)}, D_{0}^{(2, \ldots, 2 m)}\right], \\
x_{2 k i+1}= & a_{1} x_{2 k i-1}+b_{1} f_{\lambda}\left(x_{2 k i}\right) \\
= & a_{1} x_{2 k i-1} \in\left(E_{j-i}^{(3, \ldots, 2 l-1)}, E_{j-i}^{(3, \ldots, 2 l+1)}\right], \\
\vdots & \\
x_{2 k i+2 m-1} & a_{2 m-1} x_{2 k i+2 m-3}+b_{2 m-1} f_{\lambda}\left(x_{2 k i+2 m-2}\right) \\
= & a_{1} a_{3} \cdots a_{2 m-1} \rho^{i} x_{-1} \\
& \in\left(E_{j-i}^{(2 m+1, \ldots, 2 l-1)}, E_{j-i}^{(2 m+1, \ldots, 2 l+1)}\right], \\
= & a_{2 m} x_{2 k i+2 m-2}+b_{2 m} f_{\lambda}\left(x_{2 k i+2 m-1}\right) \\
= & a_{1} a_{3} \cdots a_{2 m} \delta^{i} \in\left(a_{2 m} D_{0}^{(-2)}, D_{0}^{(-2)}\right] \subset(0, \lambda] .
\end{aligned}
$$

(iv) Similar to (iii), by distinguishing four different cases and by induction, we have the following.

Case 1. Consider $\left(x_{2 l}, x_{2 l+1}\right) \in(\lambda,+\infty) \times(0, \lambda], i>j=0, l \geq$ $m \geq 0$.

Case 2. Consider $\left(x_{2 l+1}, x_{2 l+2}\right) \in(0, \lambda] \times(\lambda,+\infty), i>j=$ $0, m>l \geq 0$.

Case 3. Consider $\left(x_{2 k j+2 l}, x_{2 k j+2 l+1}\right) \in(\lambda,+\infty) \times(0, \lambda], i>$ $j>0, m \geq l \geq 0$.

Case 4. Consider $\left(x_{2 k j+2 l+1}, x_{2 k j+2 l+2}\right) \in(0, \lambda] \times(\lambda,+\infty), i>$ $j>0, m \geq l \geq 0$.

Note that, in view of (A.3) and (A.4), the above result handles all solutions of $(7)$ originated from $(\lambda,+\infty)^{2}$. Together with Theorems 6 and 7, we have taken care of all the cases where $\left(x_{-2}, x_{-1}\right) \in \Omega$. Since $\Psi \cup \Omega \cup(-\infty, 0]^{2}$ is the complement of the $\mathbb{R}^{2}$, Theorems 1 and 5-7 can be used to take care of all possible solutions of (7).

\section{The Case $0<\lambda<1$}

In this section, we assume that $0<\lambda<1$ and we set $(p, q]=\emptyset$ if $p \geq q$. We continue to use those notations in the case where $\lambda=1$.

Lemma 9. Let $0<\lambda<1$. If $\left\{\left\langle x_{2 k n}\right\rangle\right\}_{n=0}^{\infty}$ is a solution of (7) with $\left(x_{-2}, x_{-1}\right) \in \mathbb{R}^{2} \backslash(-\infty, 0]^{2}$, then there exist integers $i \in \mathbb{N}$ and $m \in\{0,1, \ldots, 2 k-2\}$ such that $\left(x_{2 k i+m}, x_{2 k i+m+1}\right) \in(0, \lambda] \times$ $(\lambda,+\infty) \cup(\lambda,+\infty) \times(0, \lambda]$.

Proof. There are seven cases.

(i) If $\left(x_{-2}, x_{-1}\right) \in(0, \lambda] \times(\lambda,+\infty)$ or $\left(x_{-2}, x_{-1}\right) \in$ $(\lambda,+\infty) \times(0, \lambda]$, then, by $(7)$, we may easily see that $\left(x_{0}, x_{1}\right) \in$ $(0, \lambda] \times(\lambda,+\infty)$ or $\left(x_{0}, x_{1}\right) \in(\lambda,+\infty) \times(0, \lambda]$.

(ii) Suppose $\left(x_{-2}, x_{-1}\right) \in(0, \lambda]^{2}$. If $x_{2 k i+m} \in(0, \lambda]$ for all $i \in \mathbb{N}, m \in\{0,1, \ldots, 2 k-1\}$, then $x_{2 k i+m}=a_{m} x_{2 k i+m-2}+b_{m}$ for all $i \in \mathbb{N}, m \in\{0,1, \ldots, 2 k-1\}$. Thus, $\lim _{i \rightarrow \infty} x_{2 k i+m}=1>\lambda$, which is a contradiction. Therefore, there exists $j \in \mathbb{N}$ such that

$$
\begin{gathered}
x_{2 k j} \in(\lambda,+\infty), x_{0}, x_{1}, \ldots, x_{2 k-1}, x_{2 k}, x_{2 k+1}, \ldots, \\
x_{4 k-1}, \ldots, x_{2 k j-2}, x_{2 k j-1} \in(0, \lambda] .
\end{gathered}
$$

(iii) Suppose $\left(x_{-2}, x_{-1}\right) \in(\lambda,+\infty) \times(\lambda,+\infty)$. If $x_{2 k i+m} \in$ $(\lambda,+\infty)$ for all $i \in \mathbb{N}, m \in\{0,1, \ldots, 2 k-1\}$, then $x_{2 k i+m}=$ $a_{m} x_{2 k i+m-2}$, for all $i \in \mathbb{N}, m \in\{0,1, \ldots, 2 k-1\}$. Thus $\lim _{j \rightarrow \infty} x_{2 k j+m}=0$, which is a contradiction. Therefore, there exists $j \in \mathbb{N}$ such that

$$
\begin{gathered}
x_{2 k j} \in(0, \lambda], x_{0}, x_{1}, \ldots, x_{2 k-1}, x_{2 k}, x_{2 k+1}, \ldots, \\
x_{4 k-1}, \ldots, x_{2 k j-2}, x_{2 k j-1}>\lambda .
\end{gathered}
$$

(iv) If $\left(x_{-2}, x_{-1}\right) \in(-\infty, 0] \times(0, \lambda]$, then, in view of (A.15), there exist $i \in \mathbb{N}, r \in\{0,1, \ldots, k-1\}$ such that $x_{-2} \in$ $\left(G_{i}^{(0,2, \ldots, 2 r)}, L_{i}^{(0,2, \ldots, 2 r)}\right] \cup\left(L_{i}^{(0,2, \ldots, 2 r)}, G_{i}^{(0,2, \ldots, 2 r-2)}\right]$. Furthermore, if $x_{-2} \in\left(G_{i}^{(0,2, \ldots, 2 r)}, L_{i}^{(0,2, \ldots, 2 r)}\right]$, we distinguish two cases. 
Case 1. Consider $i=0, r \geq 0$. Then,

$$
\begin{aligned}
x_{0}= & a_{0} x_{-2}+b_{0} f_{\lambda}\left(x_{-1}\right) \\
& =a_{0} x_{-2}+b_{0} \in\left(G_{0}^{(2, \ldots, 2 r)}, L_{0}^{(2, \ldots, 2 r)}\right], \\
x_{1} & =a_{1} x_{-1} \in(0, \lambda], \\
& \vdots \\
x_{2 r}= & a_{2 r} x_{2 r-2}+b_{2 r} \in\left(G_{0}^{(-2)}, L_{0}^{(-2)}\right]=(0, \lambda], \\
x_{2 r+1} & =a_{1} \cdots a_{2 r+1} x_{2 r-1}+b_{2 r+1} \in(0,+\infty) .
\end{aligned}
$$

Case 2. Consider $i>0, r \geq 0$. Then,

$$
\begin{aligned}
x_{0} & =a_{0} x_{-2}+b_{0} f_{\lambda}\left(x_{-1}\right) \\
& =a_{0} x_{-2}+b_{0} \in\left(G_{i}^{(2, \ldots, 2 r)}, L_{i}^{(2, \ldots, 2 r)}\right], \\
x_{1} & =a_{1} x_{-1} \in(0, \lambda], \\
& \vdots \\
x_{2 k i+2 r} & =a_{2 r} x_{2 k i+2 r-2}+b_{2 r} \in\left(G_{0}^{(-2)}, L_{0}^{(-2)}\right]=(0, \lambda], \\
x_{2 k i+2 r+1} & =a_{2 r+1} x_{2 k i+2 r-1}+b_{2 r+1} \in(0,+\infty) .
\end{aligned}
$$$$
\text { Next, suppose } x_{-2} \in\left(L_{i}^{(0,2, \ldots, 2 r)}, G_{i}^{(0,2, \ldots, 2 r-2)}\right] \neq \emptyset \text {. }
$$

Case 1. Consider $i=0, r \geq 0$. From $L_{0}^{(0,2, \ldots, 2 r)} \leq G_{0}^{(0,2, \ldots, 2 r-2)}$, we may get $0<\lambda<1-a_{2 r}=b_{2 r}$. Then,

$$
\begin{aligned}
x_{0} & =a_{0} x_{-2}+b_{0} f_{\lambda}\left(x_{-1}\right) \\
& =a_{0} x_{-2}+b_{0} \in\left(L_{0}^{(2, \ldots, 2 r)}, G_{0}^{(2, \ldots, 2 r-2)}\right], \\
x_{1} & =a_{1} x_{-1} \in(0, \lambda], \\
& \vdots \\
x_{2 r} & =a_{2 r} x_{2 r-2}+b_{2 r} \in\left(L_{0}^{(-2)}, a_{2 r} G_{0}^{(-2)}+b_{2 r}\right]=\left(\lambda, b_{2 r}\right], \\
x_{2 r+1} & =a_{2 r+1} x_{2 r-1} \in(0, \lambda] .
\end{aligned}
$$

Case 2. Consider $i>0, r \geq 0$. From $L_{i}^{(0,2, \ldots, 2 r)} \leq G_{i}^{(0,2, \ldots, 2 r-2)}$, we may get $0<\lambda<1-a_{2 r}=b_{2 r}$. Then,

$$
\begin{aligned}
x_{0}= & a_{0} x_{-2}+b_{0} f_{\lambda}\left(x_{-1}\right) \\
= & a_{0} x_{-2}+b_{0} \in\left(L_{i}^{(2, \ldots, 2 r)}, G_{i}^{(2, \ldots, 2 r-2)}\right], \\
x_{1}= & a_{1} x_{-1} \in(0, \lambda], \\
\vdots & \\
x_{2 k i+2 r}= & a_{2 r} x_{2 k i+2 r-2}+b_{2 r} \\
& \in\left(L_{0}^{(-2)}, a_{2 r} G_{0}^{(-2)}+b_{2 r}\right]=\left(\lambda, b_{2 r}\right], \\
x_{2 k i+2 r+1} & a_{2 r+1} x_{2 k i+2 r-1} \in(0, \lambda] .
\end{aligned}
$$

Theorem 10. Let $0<\lambda<1$. Then, any solution $\left\{\left\langle x_{2 k n}\right\rangle\right\}_{n=0}^{\infty}$ of (7) tends asymptotically to the 2 -cycle $\langle 0,1\rangle$ or $\langle 1,0\rangle$.

Proof. In view of Lemma 9, we may assume without loss of generality that $0<x_{-2}<\lambda, x_{-1}>\lambda$. Then, by (7),

$$
\begin{aligned}
& x_{0}=a_{0} x_{-2}+b_{0} f_{\lambda}\left(x_{-1}\right)=a_{0} x_{-2} \in\left(0, a_{0} \lambda\right] \text {, } \\
& x_{1}=a_{1} x_{-1}+b_{1} f_{\lambda}\left(x_{0}\right)=a_{1} x_{-1}+b_{1}>\lambda, \\
& x_{2 k-2}=a_{2 k-2} x_{2 k-4}+b_{2 k-2} f_{\lambda}\left(x_{2 k-3}\right) \\
& =\delta x_{-2}+1-\delta \in(0, \delta \lambda] \text {, } \\
& x_{2 k-1}=a_{2 k-1} x_{2 k-3}+b_{2 k-1} f_{\lambda}\left(x_{2 k-2}\right) \\
& =\delta x_{-1}+1-\delta>\lambda, \\
& x_{2 k}=a_{0} x_{2 k-2}+b_{0} f_{\lambda}\left(x_{2 k-1}\right)=a_{0} \delta x_{-2} \in\left(0, a_{0} \delta \lambda\right] \text {, } \\
& x_{2 k+1}=a_{1} x_{2 k-1}+b_{1} f_{\lambda}\left(x_{2 k}\right)=a_{1} \rho x_{-1}+1-a_{1} \rho>\lambda \text {, } \\
& x_{4 k-2}=a_{2 k-2} x_{4 k-4}+b_{2 k-2} f_{\lambda}\left(x_{4 k-3}\right) \\
& =\delta^{2} x_{-2} \in\left(0, \delta^{2} \lambda\right] \\
& x_{4 k-1}=a_{2 k-1} x_{4 k-3}+b_{2 k-1} f_{\lambda}\left(x_{4 k-2}\right) \\
& =\rho^{2} x_{-1}+1-\rho^{2}>\lambda,
\end{aligned}
$$

and by induction, we have $\left(x_{2 k n+2 m}, x_{2 k n+2 m+1}\right) \in(0, \lambda] \times$ $(\lambda,+\infty)$ for $n \in \mathbb{N}, m \in\{0,1, \ldots, k-1\}$. Therefore, $\left\{\left\langle x_{2 k n}\right\rangle\right\}_{n=0}^{\infty}$ tends towards the limit 2 -cycle $\langle 0,1\rangle$. The proof is complete.

By reviewing Theorems 1, 3, 5-8 and 10 carefully, we may see that all solutions of (7) tend towards the limit 1cycles $\langle 0\rangle$ or $\langle 1\rangle$ or towards the limit 2 -cycles $\langle 1,0\rangle$ or $\langle 0,1\rangle$. In Theorems $1,3,5-8$, we are precise about the limit cycle for each solution from $\mathbb{R}^{2} \backslash(-\infty, 0]^{2}$, but not in Theorem 10. We can also be precise in the last case. There are more technical details, however. Hence, we refer the readers who are interested in the precise "initial vector-limit vector" relations to the Appendix.

\section{Discussions}

The results in the previous sections are stated in terms of the $2 k$-dimensional asynchronous dynamical system (7). Note that (7) is asynchronous in the sense that, given $x_{-2}$ and $x_{-1}$, the subsequent $x_{0}, x_{1}, \ldots$ are calculated one by one from the first, second, third, ... equation of (7), respectively. 
We may also regard the original equation (5) as the following 2-dimensional asynchronous system:

$$
\begin{array}{r}
x_{n}=a_{n} x_{n-2}+b_{n} f_{\lambda}\left(x_{n-1}\right), \\
x_{n+1}=a_{n+1} x_{n-1}+b_{n+1} f_{\lambda}\left(x_{n}\right), \\
n=0,1,2, \ldots
\end{array}
$$

Let us say that a solution $\left\{\left(x_{n}, x_{n+1}\right)\right\}_{n=-2}^{\infty}$ of (39) approaches a limit 1-cycle $(\alpha, \alpha)$ if $\lim _{n}\left(x_{n}, x_{n+1}\right)=(\alpha, \alpha)$ and a limit 2-cycle $(\alpha, \beta)$ if $\alpha \neq \beta$ and $\lim _{n}\left(x_{2 n}, x_{2 n+1}\right)=(\alpha, \beta)$. Let us further say that a solution $\left\{\left(x_{n}, x_{n+1}\right)\right\}_{n=-2}^{\infty}$ of (39) eventually falls into a region $\Upsilon$ if $\left(x_{n}, x_{n+1}\right) \in \Upsilon$ for all large $n$ and eventually alternates between two regions $\Upsilon_{1}$ and $\Upsilon_{2}$ if $\left(x_{2 n}, x_{2 n+1}\right) \in \Upsilon_{1}$ and $\left(x_{2 n+1}, x_{2 n+2}\right) \in \Upsilon_{2}$ for all large $n$.

Then, we may restate the previous theorems as follows.

(i) The vectors $(0,0),(1,1),(0,1)$, and $(1,0)$ form the corners of a square in the plane.

(ii) A solution $\left\{\left(x_{n}, x_{n+1}\right)\right\}_{n=-2}^{\infty}$ of (39) with $\left(x_{-2}, x_{-1}\right)$ in the nonpositive orthant $(-\infty, 0]^{2}$ is nonpositive and tends towards the limit 1-cycle $(0,0)$.

(iii) Suppose $\lambda>1$. Then, a solution $\left\{\left(x_{n}, x_{n+1}\right)\right\}_{n=-2}^{\infty}$ of (39) with $\left(x_{-2}, x_{-1}\right) \in \mathbb{R}^{2} \backslash(-\infty, 0]^{2}$ will tend towards the limit 1-cycle $(1,1)$.

(iv) Suppose $0<\lambda<1$. Then, a solution $\left\{\left(x_{n}, x_{n+1}\right)\right\}_{n=-2}^{\infty}$ of (39) with $\left(x_{-2}, x_{-1}\right) \in \mathbb{R}^{2} \backslash(-\infty, 0]^{2}$ will tend towards the limit 2-cycle $(0,1)$ or $(1,0)$.

(v) Suppose $\lambda=1$. Then, a solution $\left\{\left(x_{n}, x_{n+1}\right)\right\}_{n=-2}^{\infty}$ of (39) with $\left(x_{-2}, x_{-1}\right) \in \Psi$ will eventually fall into $(0, \lambda] \times(0, \lambda]$ and approach the limit 1-cycle $(1,1)$.

(vi) Suppose $\lambda=1$. Then, a solution $\left\{\left(x_{n}, x_{n+1}\right)\right\}_{n=-2}^{\infty}$ of (39) with $\left(x_{-2}, x_{-1}\right) \in \Omega$ will eventually alternate between $(0, \lambda] \times(\lambda,+\infty)$ and $(\lambda,+\infty) \times(0, \lambda]$ and approach the limit 2-cycle $(0,1)$ or $(1,0)$.

Since we have obtained a complete set of asymptotic criteria, we may deduce (bifurcation) results such as the following. If it $0<\lambda<1$, then all solutions $\left\{\left(x_{n}, x_{n+1}\right)\right\}_{n=-2}^{\infty}$ which originated from the positive orthant approach a limit 2 -cycle; if $\lambda>1$, then all solutions that originated from the positive orthant tend towards the limit 1-cycles $(1,1)$; if $\lambda=1$, then all solutions originated from the positive orthant tend towards the limit 1-cycle $(1,1)$ or 2 -cycles $(1,0)$ or $(0,1)$. Roughly, the above statements show that when the threshold parameter $\lambda$ is a relatively small positive parameter, all solutions from the positive orthant tend towards a limit 2-cycle; when the threshold parameter reaches the critical value 1 , some of these solutions drift away and tend towards a limit 1-cycle, and when $\lambda$ drifts beyond the critical value, all solutions tend towards the limit 1 -cycle $(1,1)$. Such an observation seems to appear in many natural processes and hence our model may be used to explain such phenomena. It is also expected that when a group of neural units interact with each other in a network where each unit is governed by evolutionary laws of the form (39), complex but manageable analytical results can be obtained. These are left for further studies.
We remark that the precise region from which each type of solution originates can also be given (by implementing a simple computer program).

Our proofs also indicate that more general multiple neuron recurrent McCulloch-Pitts type neural networks possess similar behavior. However, the derivations may involve more delicate combinatorial arguments and are left for further studies.

\section{Appendix}

We let

$$
\begin{aligned}
& D_{i}^{(-2)}=\frac{\lambda}{\delta^{i}}, \quad E_{i}^{(-1)}=\frac{\lambda}{\rho^{i}}, \\
& D_{i}^{\left(j_{0}, j_{1}, \ldots, j_{m}\right)}=\frac{\lambda}{a_{j_{0}} a_{j_{1}} \cdots a_{j_{m}} \delta^{i}}, \\
& E_{i}^{\left(j_{0}, j_{1}, \ldots, j_{m}\right)}=\frac{\lambda}{a_{j_{0}} a_{j_{1}} \cdots a_{j_{m}} \rho^{i}} .
\end{aligned}
$$

Then,

$$
\begin{gathered}
D_{0}^{(-2)}=E_{0}^{(-1)}=\lambda, \\
E_{i}^{(1,3, \ldots, 2 k-1)}=E_{i+1}^{(-1)}, \\
D_{i}^{(0,2, \ldots, 2 k-2)}=D_{i+1}^{(-2)}, \\
E_{i}^{\left(j_{0}, j_{1}, \ldots, j_{m}\right)}<E_{i}^{\left(j_{0}, j_{1}, \ldots, j_{m+1}\right)}, \\
D_{i}^{\left(j_{0}, j_{1}, \ldots, j_{m}\right)}<D_{i}^{\left(j_{0}, j_{1}, \ldots, j_{m+1}\right)}, \\
\lim _{i \rightarrow \infty} D_{i}^{\left(j_{0}, j_{1}, \ldots, j_{m}\right)}=\lim _{i \rightarrow \infty} E_{i}^{\left(j_{0}, j_{1}, \ldots, j_{m}\right)}=+\infty .
\end{gathered}
$$

Thus,

$$
\begin{aligned}
& (\lambda,+\infty)=\bigcup_{i=0}^{\infty} \bigcup_{m=0}^{k-1}\left(D_{i}^{(0,2, \ldots, 2 m-2)}, D_{i}^{(0,2, \ldots, 2 m)}\right], \\
& (\lambda,+\infty)=\bigcup_{i=0}^{\infty} \bigcup_{m=0}^{k-1}\left(E_{i}^{(1,3, \ldots, 2 m-1)}, E_{i}^{(1,3, \ldots, 2 m+1)}\right] .
\end{aligned}
$$

We assume $\lambda>1$. Set

$$
\begin{gathered}
A_{i}^{(-1)}=\frac{\lambda-\left(1-\rho^{i}\right)}{\rho^{i}}, \\
B_{i}^{(-2)}=\frac{\lambda-\left(1-\delta^{i}\right)}{\delta^{i}}, \\
A_{i}^{\left(j_{0}, j_{1}, \ldots, j_{m}\right)}=\frac{\lambda-\left(1-a_{j_{0}} a_{j_{1}} \cdots a_{j_{m}} \rho^{i}\right)}{j_{0} j_{1} \cdots j_{m} \rho^{i}}, \\
B_{i}^{\left(j_{0}, j_{1}, \ldots, j_{m}\right)}=\frac{\lambda-\left(1-a_{j_{0}} a_{j_{1}} \cdots a_{j_{m}} \delta^{i}\right)}{j_{0} j_{1} \cdots j_{m} \delta^{i}} .
\end{gathered}
$$


Then,

$$
\begin{gathered}
A_{0}^{(-1)}=B_{0}^{(-2)}=\lambda, \\
A_{i}^{(1,3, \ldots, 2 k-1)}=A_{i+1}^{(-1)}, \\
B_{i}^{(0,2, \ldots, 2 k-2)}=B_{i+1}^{(-2)}, \\
A_{i}^{\left(j_{0}, j_{1}, \ldots, j_{m}\right)}<A_{i}^{\left(j_{0}, j_{1}, \ldots, j_{m+1}\right)}, \\
B_{i}^{\left(j_{0}, j_{1}, \ldots, j_{m}\right)}<B_{i}^{\left(j_{0}, j_{1}, \ldots, j_{m+1}\right)}, \\
\lim _{i \rightarrow \infty} A_{i}^{\left(j_{0}, j_{1}, \ldots, j_{m}\right)}=\lim _{i \rightarrow \infty} B_{i}^{\left(j_{0}, j_{1}, \ldots, j_{m}\right)}=+\infty .
\end{gathered}
$$

Thus,

$$
\begin{aligned}
& (\lambda,+\infty)=\bigcup_{i=0}^{\infty} \bigcup_{m=0}^{k-1}\left(A_{i}^{(1,3, \ldots, 2 m-1)}, A_{i}^{(1,3, \ldots, 2 m+1)}\right], \\
& (\lambda,+\infty)=\bigcup_{i=0}^{\infty} \bigcup_{m=0}^{k-1}\left(B_{i}^{(0,2, \ldots, 2 m-2)}, B_{i}^{(0,2, \ldots, 2 m)}\right] .
\end{aligned}
$$

We assume $0<\lambda<1$. Set

$$
\begin{gathered}
G_{i}^{(-2)}=-\frac{1-\delta^{i}}{\delta^{i}}, \quad H_{i}^{(-1)}=-\frac{1-\rho^{i}}{\rho^{i}}, \\
L_{i}^{(-2)}=\frac{\lambda-\left(1-\delta^{i}\right)}{\delta^{i}}, \quad M_{i}^{(-1)}=\frac{\lambda-\left(1-\rho^{i}\right)}{\rho^{i}}, \\
G_{i}^{\left(j_{0}, j_{1}, \ldots, j_{m}\right)}=-\frac{1-a_{j_{0}} a_{j_{1}} \cdots a_{j_{m}} \delta^{i}}{a_{j_{0}} a_{j_{1}} \cdots a_{j_{m}} \delta^{i}} \\
L_{i}^{\left(j_{0}, j_{1}, \ldots, j_{m}\right)}=\frac{\lambda-\left(1-a_{j_{0}} a_{j_{1}} \cdots a_{j_{m}} \delta^{i}\right)}{a_{j_{0}} a_{j_{1}} \cdots a_{j_{m}} \delta^{i}} \\
j_{0}, j_{1}, \ldots, j_{m} \in\{0,2, \ldots, 2 k-2\} \\
H_{i}^{\left(j_{0}, j_{1}, \ldots, j_{m}\right)}=-\frac{1-a_{j_{0}} a_{j_{1}} \cdots a_{j_{m}} \rho^{i}}{a_{j_{0}} a_{j_{1}} \cdots a_{j_{m}} \rho^{i}} \\
M_{i}^{\left(j_{0}, j_{1}, \ldots, j_{m}\right)}=\frac{\lambda-\left(1-a_{j_{0}} a_{j_{1}} \cdots a_{j_{m}} \rho^{i}\right)}{a_{j_{0}} a_{j_{1}} \cdots a_{j_{m}} \rho^{i}} \\
j_{0}, j_{1}, \ldots, j_{m} \in\{1,3, \ldots, 2 k-1\} .
\end{gathered}
$$

Then,

$$
\begin{gathered}
G_{0}^{(-2)}=H_{0}^{(-1)}=0, \quad L_{0}^{(-2)}=M_{0}^{(-1)}=\lambda, \\
G_{i}^{(0,2, \ldots, 2 k-2)}=G_{i+1}^{(-2)}, \quad H_{i}^{(1,3, \ldots, 2 k-1)}=H_{i+1}^{(-1)}, \\
L_{i}^{(0,2, \ldots, 2 k-2)}=L_{i+1}^{(-2)}, \quad M_{i}^{(1,3, \ldots, 2 k-1)}=M_{i+1}^{(-1)}, \\
G_{i}^{\left(j_{0}, j_{1}, \ldots, j_{m}\right)}<L_{i}^{\left(j_{0}, j_{1}, \ldots, j_{m}\right)}, \\
H_{i}^{\left(j_{0}, j_{1}, \ldots, j_{m}\right)}<M_{i}^{\left(j_{0}, j_{1}, \ldots, j_{m}\right)},
\end{gathered}
$$

$$
\begin{aligned}
& \lim _{i \rightarrow \infty} G_{i}^{\left(j_{0}, j_{1}, \ldots, j_{m}\right)}=\lim _{i \rightarrow \infty} H_{i}^{\left(j_{0}, j_{1}, \ldots, j_{m}\right)}=-\infty, \\
& \lim _{i \rightarrow \infty} L_{i}^{\left(j_{0}, j_{1}, \ldots, j_{m}\right)}=\lim _{i \rightarrow \infty} M_{i}^{\left(j_{0}, j_{1}, \ldots, j_{m}\right)}=-\infty .
\end{aligned}
$$

Since

$$
\begin{aligned}
\lambda & =L_{0}^{(-2)}>L_{0}^{(0)}>L_{0}^{(0,2)}>\cdots>L_{1}^{(-2)}>\cdots \\
& >L_{m}^{(0,2, \ldots, 2 s)}>L_{m}^{(0,2, \ldots, 2 s+2)} \longrightarrow-\infty \quad(\text { as } m \longrightarrow \infty)
\end{aligned}
$$

there exist integers $p \in \mathbb{N}$ and $t \in\{0,1, \ldots, k-1\}$ such that $L_{\text {Set }}^{(0,2, \ldots, 2 t)} \leq 0$ and $L_{p}^{(0,2, \ldots, 2 t-2)}>0$.

$$
\begin{aligned}
\bar{L}_{p}^{(0,2, \ldots, 2 t)} & =\bar{L}_{p}^{(0,2, \ldots, 2 t+2)}=\cdots=\bar{L}_{p+1}^{(-2)}=0, \\
& \bar{L}_{i}^{(0,2, \ldots, 2 s)}=L_{i}^{(0,2, \ldots, 2 s)} \\
& \bar{L}_{p}^{(0,2, \ldots, 2 l)}=L_{p}^{(0,2, \ldots, 2 l)}
\end{aligned}
$$

for $i \in\{0,1, \ldots, p-1\}, s \in\{0,1, \ldots, k-1\}$, and $l \in\{0,1, \ldots, t-$ 1\}. Similarly, since

$$
\begin{aligned}
\lambda & =M_{0}^{(-1)}>M_{0}^{(1)}>M_{0}^{(1,3)}>\cdots>L_{1}^{(-1)}>\cdots \\
& >M_{m}^{(1,1, \ldots, 2 s-1)}>M_{m}^{(1,3, \ldots, 2 s+1)} \longrightarrow-\infty \quad(\text { as } m \longrightarrow \infty),
\end{aligned}
$$

there exist integers $q \in \mathbb{N}$ and $s \in\{0,1, \ldots, k-1\}$ such that $M_{q}^{(1,3, \ldots, 2 s-1)} \leq 0$ and $M_{q}^{(0,2, \ldots, 2 s+1)}>0$.

Set $\bar{M}_{q}^{(1,3, \ldots, 2 s-1)}=\bar{M}_{q}^{(1,3, \ldots, 2 s+1)}=\cdots=\bar{M}_{q+1}^{(-2)}=0$, $\bar{M}_{i}^{(1,3, \ldots, 2 s+1)}=M_{i}^{(1,3, \ldots, 2 s+1)}$, and $\bar{M}_{q}^{(1,3, \ldots, 2 l+1)}=M_{q}^{(1,3, \ldots, 2 l+1)}$ for $i \in\{0,1, \ldots, q-1\}, s \in\{0,1, \ldots, k-1\}$, and $l \in\{0,1, \ldots, s-1\}$. Then,

$$
\begin{aligned}
& (0, \lambda]=\bigcup_{i=0}^{p} \bigcup_{m=0}^{k-1}\left(\bar{L}_{i}^{(0,2, \ldots, 2 m)}, \bar{L}_{i}^{(0,2, \ldots, 2 m-2)}\right], \\
& (0, \lambda]=\bigcup_{i=0}^{q} \bigcup_{r=0}^{k-1}\left(\bar{M}_{i}^{(1,3, \ldots, 2 r+1)}, \bar{M}_{i}^{(1,3, \ldots, 2 r-1)}\right],
\end{aligned}
$$

$$
\begin{aligned}
(-\infty, 0]= & \bigcup_{i=0}^{\infty} \bigcup_{r=0}^{k-1}\left(G_{i}^{(0,2, \ldots, 2 r)}, L_{i}^{(0,2, \ldots, 2 r)}\right] \\
& \cup\left(L_{i}^{(0,2, \ldots, 2 r)}, G_{i}^{(0,2, \ldots, 2 r-2)}\right]
\end{aligned}
$$$$
(-\infty, 0]=\bigcup_{i=0}^{\infty} \bigcup_{r=0}^{k-1}\left(H_{i}^{(1,3, \ldots, 2 r+1)}, M_{i}^{(1,3, \ldots, 2 r+1)}\right]
$$ 
Furthermore, since

$$
\begin{aligned}
& G_{i}^{(0,2, \ldots, 2 r)}<\frac{\bar{L}_{p}^{(0,2, \ldots, 2 t-2)}-\left(1-a_{0} a_{2} \cdots a_{2 r} \delta^{i}\right)}{a_{0} a_{2} \cdots a_{2 r} \delta^{i}}<\cdots \\
& <\frac{\bar{L}_{p}^{(-2)}-\left(1-a_{0} a_{2} \cdots a_{2 r} \delta^{i}\right)}{a_{0} a_{2} \cdots a_{2 r} \delta^{i}}<\cdots \\
& <\frac{\bar{L}_{0}^{(-2)}-\left(1-a_{0} a_{2} \cdots a_{2 r} \delta^{i}\right)}{a_{0} a_{2} \cdots a_{2 r} \delta^{i}} \\
& =\bar{L}_{i}^{(0,2, \ldots, 2 r)} \text {, } \\
& H_{i}^{(1,3, \ldots, 2 r+1)}<\frac{\bar{M}_{q}^{(1,3, \ldots, 2 r-1)}-\left(1-a_{1} a_{3} \cdots a_{2 r+1} \rho^{i}\right)}{a_{1} a_{3} \cdots a_{2 r+1} \rho^{i}}<\cdots \\
& <\frac{\bar{M}_{q-1}^{(-1)}-\left(1-a_{1} a_{3} \cdots a_{2 r+1} \rho^{i}\right)}{a_{1} a_{3} \cdots a_{2 r+1} \rho^{i}}<\cdots \\
& <\frac{\bar{M}_{0}^{(-1)}-\left(1-a_{1} a_{3} \cdots a_{2 r+1} \rho^{i}\right)}{a_{1} a_{3} \cdots a_{2 r+1} \rho^{i}} \\
& =\bar{M}_{i}^{(1,3, \ldots, 2 r+1)} \text {, }
\end{aligned}
$$

then if we let

$$
\begin{aligned}
\Theta= & \{0,1, \ldots, p-1\} \times\{0,1, \ldots, k-1\} \\
& \cup\{p\} \times\{0,1, \ldots, t-1\}, \\
\Xi= & \{0,1, \ldots, q-1\} \times\{0,1, \ldots, k-1\} \\
& \cup\{q\} \times\{0,1, \ldots, s-1\}, \\
Q_{i, j}^{(0,2, \ldots, 2 r ; 0,2, \ldots, 2 m-2)}=\frac{\bar{L}_{j}^{(0,2, \ldots, 2 m)}-\left(1-a_{0} a_{2} \cdots a_{2 r} \delta^{i}\right)}{a_{0} a_{2} \cdots a_{2 r} \delta^{i}}, & (j, m) \in \Theta, \\
R_{i, j}^{(1,3, \ldots, 2 r+1 ; 1,3, \ldots, 2 m-1)} & \frac{\bar{M}_{j}^{(1,3, \ldots, 2 m-1)}-\left(1-a_{1} a_{3} \cdots a_{2 r+1} \rho^{i}\right)}{a_{1} a_{3} \cdots a_{2 r+1} \rho^{i}}, \\
= & \frac{(j, m) \in \Xi}{},
\end{aligned}
$$

then

$$
\begin{aligned}
& \left(G_{i}^{(0,2, \ldots, 2 r)}, L_{i}^{(0,2, \ldots, 2 r)}\right] \\
& \quad=\bigcup_{(j, m) \in \Theta}\left(Q_{i, j}^{(0,2, \ldots, 2 r ; 0,2, \ldots, 2 m)}, Q_{i, j}^{(0,2, \ldots, 2 r ; 0,2, \ldots, 2 m-2)}\right],
\end{aligned}
$$

$$
\begin{aligned}
& \left(H_{i}^{(1,3, \ldots, 2 r+1)}, M_{i}^{(1,3, \ldots, 2 r+1)}\right] \\
& =\bigcup_{(j, m) \in \Xi}\left(R_{i, j}^{(1,3, \ldots, 2 r+1 ; 1,3, \ldots, 2 m+1)}, R_{i, j}^{(1,3, \ldots, 2 r+1 ; 1,3, \ldots, 2 m-1)}\right], \\
& (-\infty, 0] \\
& =\bigcup_{i=0}^{\infty} \bigcup_{r=0}^{k-1}\left\{\bigcup_{(j, m) \in \Theta}\left(Q_{i, j}^{(0,2, \ldots, 2 r ; 0,2, \ldots, 2 m)}, Q_{i, j}^{(0,2, \ldots, 2 r ; 0,2, \ldots, 2 m-2)}\right]\right. \\
& \left.\cup\left(L_{i}^{(0,2, \ldots, 2 r)}, G_{i}^{(0,2, \ldots, 2 r-2}\right]\right\} \\
& (-\infty, 0] \\
& =\bigcup_{i=0}^{\infty} \bigcup_{r=0}^{k-1}\left\{\bigcup_{(j, m) \in \Xi}\left(R_{i, j}^{(1, \ldots, 2 r+1 ; 1, \ldots, 2 m+1)}, R_{i, j}^{(1, \ldots, 2 r+1 ; 1, \ldots, 2 m-1)}\right]\right. \\
& \left.\cup\left(M_{i}^{(1,3, \ldots, 2 r+1)}, H_{i}^{(1,3, \ldots, 2 r-1)}\right]\right\} .
\end{aligned}
$$

Furthermore,

$$
\begin{array}{r}
(0, \lambda]=\left(\bigcup_{(i, m) \in \Theta}\left(\frac{\bar{L}_{i}^{(0, \ldots, 2 m)}}{a_{0} \cdots a_{2 s} \delta^{j}}, \frac{\bar{L}_{i}^{(0, \ldots, 2 m-2)}}{a_{0} \cdots a_{2 s} \delta^{j}}\right]\right) \cap(0, \lambda], \\
j \in \mathbb{N}, \quad s \in\{0,1, \ldots, k-1\},
\end{array}
$$

$$
\begin{array}{r}
\left(\bigcup_{i, m \in \Xi}\left(\frac{\bar{M}_{i}^{(1, \ldots, 2 m+1)}}{a_{1} a_{3} \cdots a_{2 r+1} \rho^{j}}, \frac{\bar{M}_{i}^{(1, \ldots, 2 m-1)}}{a_{1} \cdots a_{2 r+1} \rho^{j}}\right]\right) \cap(0, \lambda], \\
j \in \mathbb{N}, \quad r \in\{0,1, \ldots, k-1\},
\end{array}
$$

$(-\infty, 0]$

$$
\begin{array}{r}
=\bigcup_{i=0}^{\infty} \bigcup_{r=0}^{k-1}\left\{\bigcup _ { ( j , m ) \in \Theta } \left(\left(\frac{Q_{i, j}^{(0, \ldots, 2 r ; 0,2, \ldots, 2 m)}}{a_{0} \cdots a_{2 \xi} \delta^{n}},\right.\right.\right. \\
\left.\frac{Q_{i, j}^{(0, \ldots, 2 r ; 0, \ldots, 2 m-2)}}{a_{0} \cdots a_{2 \xi} a_{0} \cdots a_{2 \eta} \delta^{n}}\right]
\end{array}
$$$$
\cup\left(\frac{L_{i}^{(0, \ldots, 2 r)}}{a_{0} \cdots a_{2 \xi} a_{0} \cdots a_{2 \eta} \delta^{n}},\right.
$$$$
\left.\left.\left.\frac{G_{i}^{(0, \ldots, 2 r-2)}}{a_{0} \cdots a_{2 \xi} a_{0} \cdots a_{2 \eta} \delta^{n}}\right]\right)\right\},
$$

$\xi, \eta, n \in \mathbb{N}$ 
$(-\infty, 0]$

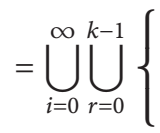

$$
\bigcup_{(j, m) \in \Xi}(
$$

$$
\begin{gathered}
\left(\frac{R_{i, j}^{(1, \ldots, 2 r+1 ; 1, \ldots, 2 m+1)}}{a_{1} \cdots a_{2 \xi+1} a_{1} \cdots a_{2 \eta+1} \rho^{n}},\right. \\
\left.\frac{R_{i, j}^{(1, \ldots, 2 r+1 ; 1, \ldots, 2 m-1)}}{a_{1} \cdots a_{2 \xi+1} a_{1} \cdots a_{2 \eta+1} \rho^{n}}\right] \\
\cup\left(\frac{M_{i}^{(1, \ldots, 2 r+1)}}{a_{1} \cdots a_{2 \xi+1} a_{1} \cdots a_{2 \eta+1} \rho^{n}},\right. \\
\left.\left.\left.\frac{H_{i}^{(1, \ldots, 2 r-1)}}{a_{1} \cdots a_{2 \xi+1} a_{1} \cdots a_{2 \eta+1} \rho^{n}}\right]\right)\right\},
\end{gathered}
$$

$\xi, \eta, n \in \mathbb{N}$.

(A.20)

We also note that

$$
\begin{aligned}
& \frac{\bar{L}_{p}^{(0, \ldots, 2 t)}}{a_{0} \cdots a_{2 n} a_{0} \cdots a_{2 t} \delta^{\tau+i}}=0, \\
& \frac{\bar{M}_{q}^{(1, \ldots, 2 s+1)}}{a_{1} \cdots a_{2 n+1} a_{1} \cdots a_{2 s+1} \rho^{\tau+i}}=0, \\
& \frac{\bar{L}_{0}^{(-2)}}{a_{0} \cdots a_{2 n} a_{0} \cdots a_{2 t} \delta^{\tau+i}}=\frac{\lambda}{a_{0} \cdots a_{2 n} a_{0} \cdots a_{2 t} \delta^{\tau+i}} \\
& >\frac{\lambda}{a_{0} \cdots a_{2 n} a_{1} \cdots a_{2 t-2} \delta^{\tau+i}}>\cdots \\
& >\frac{\lambda}{\delta^{\tau+i}}>\cdots>D_{0}^{(-2)}=\lambda \text {, } \\
& \frac{\bar{M}_{0}^{(-1)}}{a_{1} \cdots a_{2 n+1} a_{1} \cdots a_{2 s+1} \rho^{\tau+i}}=\frac{\lambda}{a_{1} \cdots a_{2 n+1} a_{1} \cdots a_{2 s+1} \rho^{\tau+i}} \\
& >\frac{\lambda}{a_{1} \cdots a_{2 n+1} a_{1} \cdots a_{2 s-1} \rho^{\tau+i}}>\cdots \\
& >\frac{\lambda}{\rho^{\tau+i}}>\cdots>E_{0}^{(-1)}=\lambda \text {. }
\end{aligned}
$$

Therefore,

$$
\begin{gathered}
(\lambda,+\infty) \\
=\bigcup_{\tau=0}^{\infty} \bigcup_{n=0}^{k-1} \bigcup_{(i, m) \in \Theta}\left(\frac{\bar{L}_{i}^{(0, \ldots, 2 m)}}{a_{0} \cdots a_{2 n} a_{0} \cdots a_{2 r} \delta^{\tau+i}},\right. \\
\left.\frac{\bar{L}_{i}^{(0,2, \ldots, 2 m)}}{a_{0} \cdots a_{2 n} a_{0} \cdots a_{2 r} \rho^{\tau+i}}\right] \\
\cap\left(D_{\tau}^{(0, \ldots, 2 n-2)}, D_{\tau}^{(0, \ldots, 2 n)}\right],
\end{gathered}
$$

$(\lambda,+\infty)$

$$
\begin{gathered}
=\bigcup_{\tau=0}^{\infty} \bigcup_{n=0}^{k-1}\left(\bigcup _ { ( i , m ) \in \Xi } \left(\frac{\bar{M}_{i}^{(1, \ldots, 2 m+1)}}{a_{1} \cdots a_{2 n+1} a_{1} \cdots a_{2 r+1} \rho^{\tau+i}},\right.\right. \\
\left.\frac{\bar{M}_{i}^{(1, \ldots, 2 m-1)}}{a_{1} \cdots a_{2 n+1} a_{1} \cdots a_{2 r+1} \rho^{\tau+i}}\right] \\
\left.\cap\left(E_{\tau}^{(1, \ldots, 2 n-1)}, E_{\tau}^{(1, \ldots, 2 n+1)}\right]\right) .
\end{gathered}
$$

Lemma A.1. A solution $\left\{\left\langle x_{2 k n}\right\rangle\right\}_{n=0}^{\infty}$ of (7) with $\left(x_{-2}, x_{-1}\right) \in$ $(0, \lambda] \times(\lambda,+\infty)\left(\right.$ or $\left.\left(x_{-2}, x_{-1}\right) \in(\lambda,+\infty) \times(0, \lambda]\right)$ tends towards the limit 2-cycle $\langle 0,1\rangle$ (or $\langle 1,0\rangle)$.

The proof of Lemma A.1 is the same as those discussed in the proofs of Theorems 6 and 7.

Lemma A.2. Suppose $\left\{\left\langle x_{2 k n}\right\rangle\right\}_{n=0}^{\infty}$ is a solution of (7) with $\left(x_{-2}, x_{-1}\right) \in\left(D_{i}^{(0,2, \ldots, 2 m-2)}, D_{i}^{(0,2, \ldots, 2 m)}\right] \times\left(E_{i}^{(1,3, \ldots, 2 l-1)}\right.$, $\left.E_{i}^{(1,3, \ldots, 2 l+1)}\right]$, where $i, j \in\{0,1, \ldots\}$ and $m, l \in\{0,1,2, \ldots k-1\}$. Then,

(i) $\left(x_{2 k i+2 m}, x_{2 k i+2 m+1}\right) \in(0, \lambda] \times(\lambda,+\infty)$ for $0 \leq i=j$, $0 \leq m \leq l$;

(ii) $\left(x_{2 k i+2 l}, x_{2 k i+2 l+1}\right) \in(\lambda,+\infty) \times(0, \lambda)$ for $0 \leq i=j$, $0 \leq l<m$;

(iii) $\left(x_{2 k i+2 m}, x_{2 k i+2 m+1}\right) \in(0, \lambda] \times(\lambda,+\infty)$ for $0 \leq i<j$;

(iv) $\left(x_{2 k j+2 l}, x_{2 k j+2 l+1}\right) \in(\lambda,+\infty) \times(0, \lambda]$ for $0 \leq j<i$.

Lemma A.3. Suppose $\left\{\left\langle x_{2 k n}\right\rangle\right\}_{n=0}^{\infty}$ is a solution of (7) with $\left(x_{-2}, x_{-1}\right) \in\left(\bar{L}_{i}^{(0,2, \ldots, 2 m)}, \bar{L}_{i}^{(0,2, \ldots, 2 m-2)}\right] \times\left(\bar{M}_{j}^{(1, \ldots, 2 l+1)}, \bar{M}_{j}^{(1, \ldots, 2 l-1)}\right]$, where $i, j \in\{0,1, \ldots\}$ and $m, l \in\{0,1,2, \ldots, k-1\}$. Then,
(i) $\left(x_{2 k i+2 m}, x_{2 k i+2 m+1}\right) \in(\lambda,+\infty) \times(0, \lambda]$ for $0 \leq i=j$, $0 \leq m \leq l$;
(ii) $\left(x_{2 k i+2 l}, x_{2 k i+2 l+1}\right) \in(0, \lambda] \times(\lambda,+\infty)$ for $0 \leq i=j$, $0 \leq l<m$;
(iii) $\left(x_{2 k i+2 m}, x_{2 k i+2 m+1}\right) \in(\lambda,+\infty) \times(0, \lambda]$ for $0 \leq i<j$;
(iv) $\left(x_{2 k j+2 l}, x_{2 k j+2 l+1}\right) \in(0, \lambda] \times(\lambda,+\infty)$ for $0 \leq j<i$.

Lemma A.4. Suppose $0<\lambda<1$ and $\left\{\left\langle x_{2 k n}\right\rangle\right\}_{n=0}^{\infty}$ is a solution of $(7)$ with $\left(x_{-2}, x_{-1}\right) \in(0, \lambda] \times(-\infty, 0]$. If $\left(x_{-2}, x_{-1}\right) \in(0, \lambda] \times$ $\left(M_{i}^{(1, \ldots, 2 r+1)}, H_{i}^{(1, \ldots, 2 r-1)}\right] \neq \emptyset$, then

$$
\left(x_{2 k i+2 r}, x_{2 k i+2 r+1}\right) \in(0, \lambda] \times(\lambda, \infty) \text {. }
$$

If

$$
\begin{aligned}
& \left(x_{-2}, x_{-1}\right) \\
& \in\left(\left(\frac{\bar{L}_{l}^{(0, \ldots, 2 m)}}{a_{0} a_{2} \cdots a_{2 r} \delta^{i}}, \frac{\bar{L}_{l}^{(0, \ldots, 2 m-2)}}{a_{0} a_{2} \cdots a_{2 r} \delta^{i}}\right] \cap(0, \lambda]\right) \\
& \quad \times\left(R_{i j}^{(1,3, \ldots, 2 r+1 ; 1, \ldots, 2 n+1)}, R_{i j}^{(1,3, \ldots, 2 r+1 ; 1, \ldots, 2 n-1)}\right] \neq \emptyset,
\end{aligned}
$$


then

$$
\begin{aligned}
\left(x_{2 k i+2 r}, x_{2 k i+2 r+1}\right) \in & \left(\bar{L}_{l}^{(0, \ldots, 2 m)}, \bar{L}_{l}^{(0,2, \ldots, 2 m-2)}\right] \\
& \times\left(\bar{M}_{j}^{(1, \ldots, 2 n+1)}, \bar{M}_{j}^{(1, \ldots, 2 n-1)}\right] .
\end{aligned}
$$

Lemma A.5. Suppose $0<\lambda<1$ and $\left\{\left\langle x_{2 k n}\right\rangle\right\}_{n=0}^{\infty}$ is a solution of (7) with $\left(x_{-2}, x_{-1}\right) \in(-\infty, 0] \times(0, \lambda]$. If $\left(x_{-2}, x_{-1}\right) \in$ $\left(L_{i}^{(0, \ldots, 2 r)}, G_{i}^{(0, \ldots, 2 r-2)}\right] \times(0, \lambda] \neq \emptyset$, then

$$
\left(x_{2 k i+2 r}, x_{2 k i+2 r+1}\right) \in(\lambda, \infty) \times(0, \lambda] .
$$

If

$$
\begin{aligned}
& \left(x_{-2}, x_{-1}\right) \\
& \in\left(Q_{i j}^{(0, \ldots, 2 r ; 0, \ldots, 2 m)}, Q_{i j}^{(0, \ldots, 2 r ; 0, \ldots, 2 m-2)}\right] \\
& \quad \times\left(\left(\frac{\bar{M}_{\tau}^{(1, \ldots, 2 n+1)}}{a_{1} \cdots a_{2 r+1} \rho^{i}}, \frac{\bar{M}_{\tau}^{(1, \ldots, 2 n-1)}}{a_{1} \cdots a_{2 r+1} \rho^{i}}\right] \cap(0, \lambda]\right) \neq \emptyset,
\end{aligned}
$$

then

$$
\begin{aligned}
\left(x_{2 k i+2 r}, x_{2 k i+2 r+1}\right) \in & \left(\bar{L}_{j}^{(0, \ldots, 2 m)}, \bar{L}_{j}^{(0, \ldots, 2 m-2)}\right] \\
& \times\left(\bar{M}_{\tau}^{(1, \ldots, 2 n+1)}, \bar{M}_{\tau}^{(1, \ldots, 2 n-1)}\right] .
\end{aligned}
$$

Lemma A.6. Suppose $0<\lambda<1$ and $\left\{\left\langle x_{2 k n}\right\rangle\right\}_{n=0}^{\infty}$ is a solution of $(7)$ with $\left(x_{-2}, x_{-1}\right) \in(-\infty, 0] \times(\lambda, \infty)$. If

$$
\begin{gathered}
\left(x_{-2}, x_{-1}\right) \\
\epsilon\left(\frac{L_{j}^{(0, \ldots, 2 r)}}{a_{0} \cdots a_{2 \xi} a_{0} \cdots a_{2 \eta} \delta^{\tau+i}}, \frac{G_{j}^{(0, \ldots, 2 r-2)}}{a_{0} \cdots a_{2 \xi} a_{0} \cdots a_{2 \eta} \delta^{\tau+i}}\right] \\
\times\left(\frac{\bar{M}_{i}^{(1, \ldots, 2 m+1)}}{a_{1} a_{3} \cdots a_{2 \xi+1} a_{1} a_{3} \cdots a_{2 \eta+1} \rho^{\tau+i}},\right. \\
\left.\frac{\bar{M}_{i}^{(1, \ldots, 2 m-1)}}{a_{1} a_{3} \cdots a_{2 \xi+1} a_{1} a_{3} \cdots a_{2 \eta+1} \rho^{\tau+i}}\right] \\
\left.\cap\left(E_{\tau}^{(1, \ldots, 2 \xi-1)}, E_{\tau}^{(1, \ldots, 2 \xi+1)}\right]\right) \neq \emptyset
\end{gathered}
$$

then

$$
\begin{aligned}
& \left(x_{2 k(\tau+i)+2 \xi+2 \eta}, x_{2 k(\tau+i)+2 \xi+2 \eta+1}\right) \\
& \in\left(L_{j}^{(0, \ldots, 2 r)}, G_{j}^{(0, \ldots, 2 r-2)}\right] \\
& \quad \times\left(\bar{M}_{i}^{(1, \ldots, 2 m+1)}, \bar{M}_{i}^{(1, \ldots, 2 m-1)}\right] .
\end{aligned}
$$

If

$$
\begin{gathered}
\left(x_{-2}, x_{-1}\right) \\
\in\left(\frac{Q_{i j}^{(0, \ldots, 2 r ; 0, \ldots, 2 m)}}{a_{0} a_{2} \cdots a_{2 \xi} a_{0} \cdots a_{2 \eta} \delta^{\tau+i}}, \frac{Q_{i j}^{(0, \ldots, 2 r ; 0, \ldots, 2 m-2)}}{a_{0} \cdots a_{2 \xi} a_{0} \cdots a_{2 \eta} \delta^{\tau+i}}\right] \\
\times\left(\left(\frac{\bar{M}_{t}^{(1, \ldots, 2 s+1)}}{a_{1} \cdots a_{2 \xi+1} a_{1} \cdots a_{2 \eta+1} \rho^{i+\tau}},\right.\right. \\
\left.\frac{\bar{M}_{t}^{(1, \ldots, 2 s-1)}}{a_{1} \cdots a_{2 \xi+1} a_{1} \cdots a_{2 \eta+1} \rho^{i+\tau}}\right] \\
\left.\cap\left(E_{\tau}^{(1, \ldots, 2 \xi-1)}, E_{\tau}^{(1, \ldots, 2 \xi+1)}\right]\right) \neq \emptyset
\end{gathered}
$$

then

$$
\begin{aligned}
& \left(x_{2 k(\tau+i)+2 \xi+2 \eta}, x_{2 k(\tau+i)+2 \xi+2 \eta+1}\right) \\
& \in\left(Q_{i j}^{(0, \ldots, 2 r ; 0, \ldots, 2 m)}, Q_{i j}^{(0, \ldots, 2 r ; 0, \ldots, 2 m-2)}\right] \\
& \quad \times\left(\bar{M}_{t}^{(1, \ldots, 2 s+1)}, \bar{M}_{t}^{(1, \ldots, 2 s-1)}\right] .
\end{aligned}
$$

Lemma A.7. Suppose $0<\lambda<1$ and $\left\{\left\langle x_{2 k n}\right\rangle\right\}_{n=0}^{\infty}$ is a solution of $(7)$ with $\left(x_{-2}, x_{-1}\right) \in(\lambda, \infty) \times(-\infty, 0]$. If

$$
\begin{gathered}
\left(x_{-2}, x_{-1}\right) \\
\in\left(\left(\frac{\bar{L}_{i}^{(0, \ldots, 2 m)}}{a_{0} \cdots a_{2 \xi} a_{0} \cdots a_{2 \eta} \delta^{\tau+i}},\right.\right. \\
\left.\frac{\bar{L}_{i}^{(0,2, \ldots, 2 m-2)}}{a_{0} \cdots a_{2 \xi} a_{0} \cdots a_{2 \eta} \rho^{\tau+i}}\right] \\
\left.\cap\left(D_{\tau}^{(0, \ldots, 2 \xi-2)}, D_{\tau}^{(0, \ldots, 2 \xi)}\right]\right) \\
\times\left(\frac{M_{\tau}^{(1, \ldots, 2 r+1)}}{a_{1} \cdots a_{2 \xi+1} a_{1} \cdots a_{2 \eta+1} \rho^{\tau+i}},\right. \\
\left.\frac{H_{\tau}^{(1, \ldots, 2 r-1)}}{a_{1} \cdots a_{2 \xi+1} a_{1} \cdots a_{2 \eta+1} \rho^{\tau+i}}\right] \neq \emptyset
\end{gathered}
$$

then

$$
\begin{aligned}
& \left(x_{2 k(\tau+i)+2 \xi+2 \eta}, x_{2 k(\tau+i)+2 \xi+2 \eta+1}\right) \\
& \in\left(\bar{L}_{i}^{(0, \ldots, 2 m)}, \bar{L}_{i}^{(0,2, \ldots, 2 m-2)}\right] \\
& \quad \times\left(M_{\tau}^{(1, \ldots, 2 r+1)}, H_{\tau}^{(1, \ldots, 2 r-1)}\right] .
\end{aligned}
$$


If

$$
\begin{aligned}
& \left(x_{-2}, x_{-1}\right) \\
& \epsilon\left(\left(\frac{\bar{L}_{i}^{(0, \ldots, 2 m)}}{a_{0} \cdots a_{2 \xi} a_{0} \cdots a_{2 \eta} \delta^{\tau+i}},\right.\right. \\
& \left.\frac{\bar{L}_{i}^{(0,2, \ldots, 2 m)}}{a_{0} \cdots a_{2 \xi} a_{0} \cdots a_{2 \eta} \rho^{\tau+i}}\right] \\
& \left.\quad \cap\left(D_{\tau}^{(0, \ldots, 2 \xi-2)}, D_{\tau}^{(0, \ldots, 2 \xi)}\right]\right) \\
& \quad \times\left(\frac{R_{i, j}^{(1, \ldots, 2 \eta+1 ; 1, \ldots, 2 m+1)}}{a_{1} \cdots a_{2 \xi+1} \rho^{i+\tau}}, \frac{R_{i, j}^{(1, \ldots, 2 \eta+1 ; 1, \ldots, 2 m-1)}}{a_{1} \cdots a_{2 \xi+1} \rho^{i+\tau}}\right] \neq \emptyset,
\end{aligned}
$$

then

$$
\begin{aligned}
& \left(x_{2 k(\tau+i)+2 \xi+2 \eta}, x_{2 k(\tau+i)+2 \xi+2 \eta+1}\right) \\
& \in\left(\bar{L}_{i}^{(0, \ldots, 2 m)}, \bar{L}_{i}^{(0,2, \ldots, 2 m)}\right] \\
& \quad \times\left(R_{i, j}^{(1, \ldots, 2 m+1)}, R_{i, j}^{(1, \ldots, 2 m-1)}\right] .
\end{aligned}
$$

\section{Conflict of Interests}

The authors declare that there is no conflict of interests regarding the publication of this paper.

\section{Acknowledgment}

This study is supported by the Natural Science Foundation of China (11161049).

\section{References}

[1] H. Zhu and L. Huang, "Asymptotic behavior of solutions for a class of delay difference equation," Annals of Differential Equations, vol. 21, no. 1, pp. 99-105, 2005.

[2] Y. Chen, "All solutions of a class of difference equations are truncated periodic," Applied Mathematics Letters, vol. 15, no. 8, pp. 975-979, 2002. 


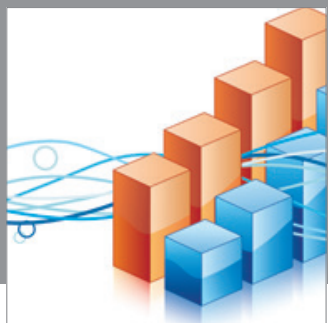

Advances in

Operations Research

mansans

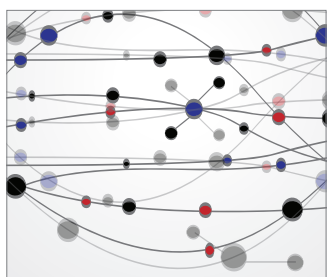

The Scientific World Journal
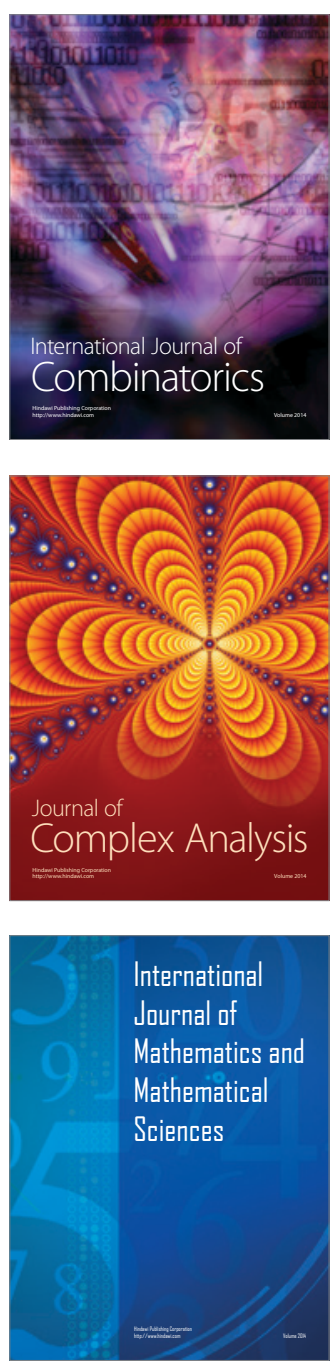
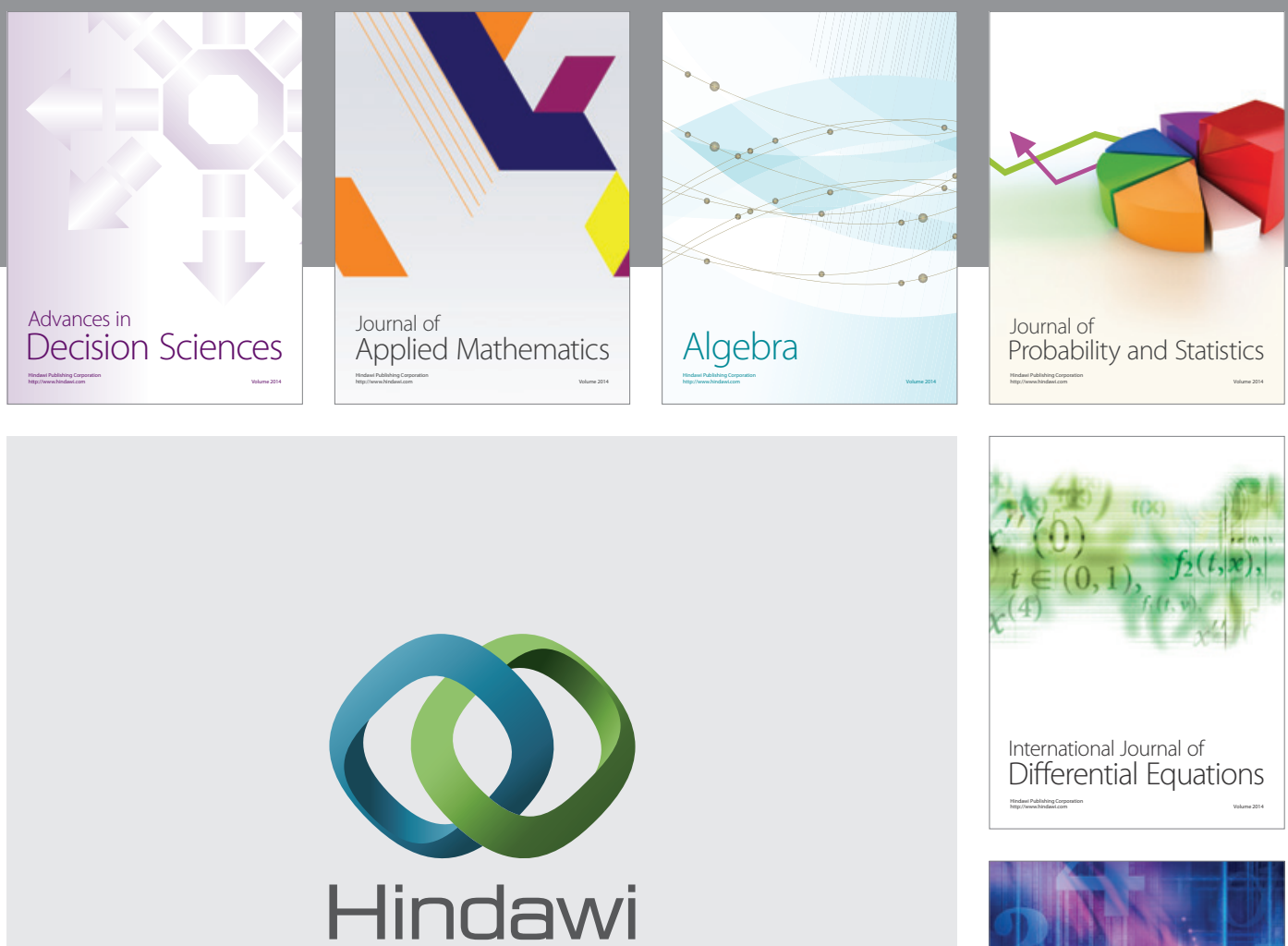

Submit your manuscripts at http://www.hindawi.com
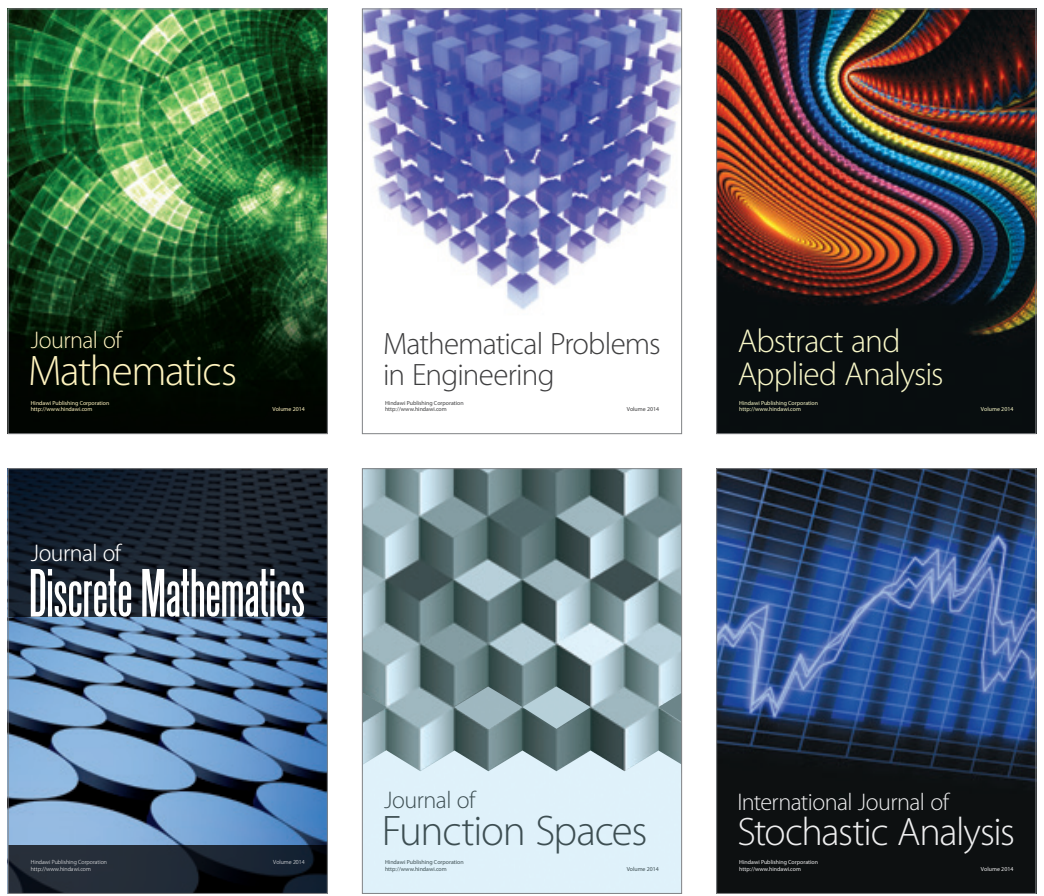

Journal of

Function Spaces

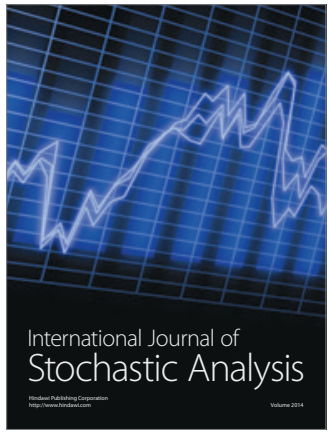

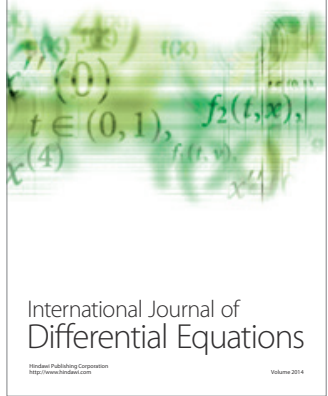
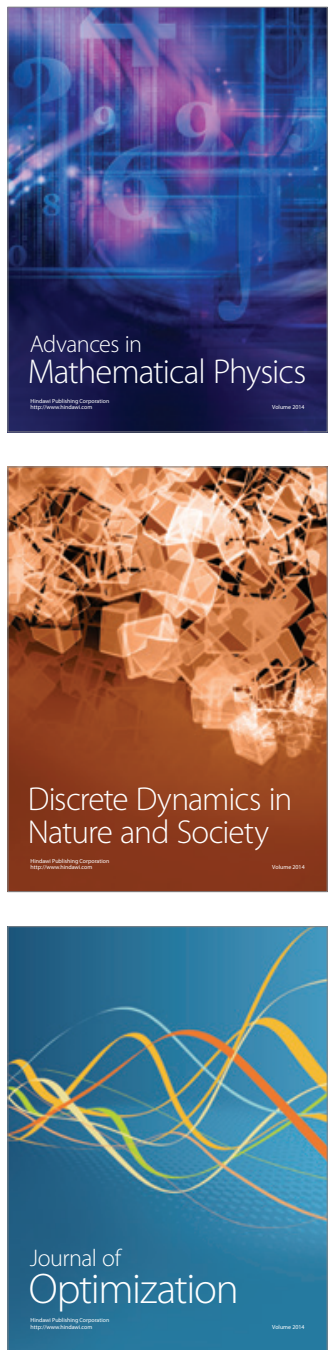